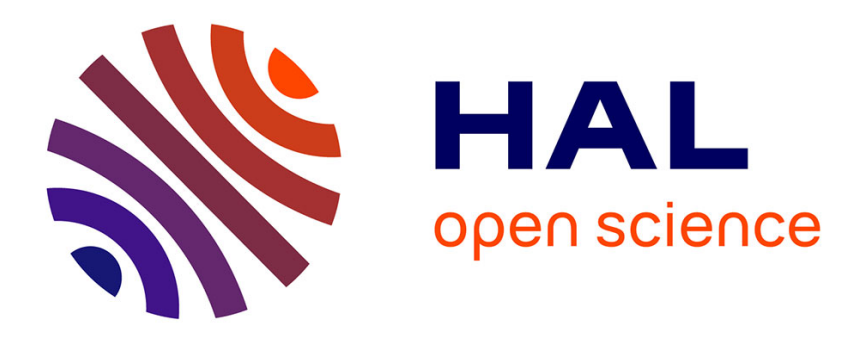

\title{
Joint distribution of a Lévy process and its running supremum
}

Laure Coutin, Waly Ngom, Monique Pontier

\section{To cite this version:}

Laure Coutin, Waly Ngom, Monique Pontier. Joint distribution of a Lévy process and its running supremum. Journal of Applied Probability, 2018, 55 (2), pp.488-512. 10.1017/jpr.2018.32 . hal01527939

\section{HAL Id: hal-01527939 \\ https://hal.science/hal-01527939}

Submitted on 26 May 2017

HAL is a multi-disciplinary open access archive for the deposit and dissemination of scientific research documents, whether they are published or not. The documents may come from teaching and research institutions in France or abroad, or from public or private research centers.
L'archive ouverte pluridisciplinaire HAL, est destinée au dépôt et à la diffusion de documents scientifiques de niveau recherche, publiés ou non, émanant des établissements d'enseignement et de recherche français ou étrangers, des laboratoires publics ou privés. 


\title{
Joint distribution of a Lévy process and its running supremum
}

\author{
Laure Coutin*Waly Ngom ${ }^{\dagger}$ Monique Pontier ${ }^{\ddagger}$ \\ February 28, 2017
}

\begin{abstract}
Let $X$ be a jump-diffusion process and $X^{*}$ its running supremum. In this paper, we first show that for any $t>0$, the law of the pair $\left(X_{t}^{*}, X_{t}\right)$ has a density with respect to Lebesgue measure and compute this one. This allows us to show that for any $t>0$, the pair formed by the random variable $X_{t}$ and the running supremum $X_{t}^{*}$ of $X$ at time $t$ can be characterized as a solution of a weakly valued-measure partial differential equation. Then we compute the marginal density of $X_{t}^{*}$ for all $t>0$.
\end{abstract}

Keywords: Lévy process, partial differential equation, running supremum process, first hitting time.

A.M.S. Classification: 60G51, 60H20, 60H99.

\section{Introduction}

Consider a Lévy process $\left(X_{t}, t \geq 0\right)$, starting from zero, which is right continuous left limited. If moreover $X$ is the sum of a drifted Brownian motion and a compound Poisson process, it is called a mixed diffusive-jump process. As any Lévy process, $X$ has stationary and independent increments and is characterized by its Laplace transform. The mixed diffusive-jump processes and the notion of first passage time (behavior of certain processes at first passage time) are very useful and widely studied.

Introducing the running supremum at time $t, X_{t}^{*}$ and the first passage $\tau_{b}$ of $X$ at level $b$, the probability $\mathbb{P}\left(X_{t} \geq a, X_{t}^{*} \geq b\right)=\mathbb{P}\left(X_{t} \geq a, \tau_{b} \leq t\right)$ for some fixed real numbers $(a, b), a \leq b$ and $b>0$, is of great importance, for example, in pricing barrier options while the logarithm of the underlying asset price is modeled by a jump-diffusion process. In this idea, Kou and Wang [5] give the explicit expression of the Laplace transform of the joint distribution of the double exponential mixed diffusive-jump process and its running supremum.

In [4], Jeanblanc et al. consider the first passage time by a diffusion at a deterministic function $h$ that depends on time and they define a function of $\tau_{h}$ and $X$ which satisfies the Fokker-Planck Equation.

\footnotetext{
*coutin@math.univ-toulouse.fr, IMT.

${ }^{\dagger}$ ngomwaly@gmail.com, IMT.

${ }^{\ddagger}$ pontier@math.univ-toulouse.fr, IMT: Institut Mathématique de Toulouse, Université Paul Sabatier, 31062 Toulouse, France.
} 
In [1], it is well noted (Theorem 2.2.9 and Exercise 2.2.10) that the $\frac{1}{2}$-stable subordinator is the first passage time of a standard Brownian motion and the inverse Gaussian subordinator is the first passage time of standard Brownian motion with a drift.

Mark Veillette and Murad S. Taqqu study in [9] the first passage time of a subordinator $D$. Since $D$ is in general non-Markovian with non-stationary and non-independent increments, they derive a partial differential equation for the Laplace transform of the $n$ - time tail distribution $\mathbb{P}\left(\tau_{t_{1}}>s_{1}, \cdots, \tau_{t_{n}}>s_{n}\right)$ where $\tau_{t_{k}}=\inf \left\{s: D_{s}>t_{k}\right\}$ for a subordinator $\left(D_{s}, s \geq 0\right)$. With this result, they give a recursive formula for multiple-time moments of the local time of a Markov process in terms of its transition density.

The authors of [2] use a partial differential equation (PDE) approach to show that the calibration of an implied volatility surface and the pricing of contingent claims can be as simple in mixed diffusive-jump framework as it is in a diffusion framework.

This work characterizes the law of the pair $U_{t}$ formed by the random variable $X_{t}$ and the running supremum $X_{t}^{*}$ of $X$ at time $t$, with a valued-measure partial differential equation and gives an explicit expression for the density function of this pair. Then the marginal density of $X_{t}^{*}$ is given. The paper is organized as follows: Section 2 provides the main result. Section 3 gives the density function of the pair formed by the random variable $X_{t}$ and its running supremum $X_{t}^{*}$ and Section 4 is devoted to the proof of the main result of Section 2. To finish, one concludes and gives some auxiliary results in Appendix.

\section{Valued measure differential equation for the joint law}

We introduce some preliminary concepts for the diffusion part: for a standard Brownian motion $W$ and a real number $m$, let be

$$
\tilde{X}_{t}=m t+W_{t}, \quad \tilde{X}_{t}^{*}=\sup _{s \leq t} \tilde{X}_{s} .
$$

In [4] page 147 , Jeanblanc et al. show that the pair $\left(\tilde{X}_{t}^{*}, \tilde{X}_{t}\right)$ has a density with respect to Lebesgue measure on $\mathbb{R}^{2}$ noted $\tilde{p}(., . ; t)$ where

$$
\tilde{p}(b, a ; t)=\frac{2(2 b-a)}{\sqrt{2 \pi t^{3}}} \exp \left[-\frac{(2 b-a)^{2}}{2 t}+m a-m^{2} \frac{t}{2}\right] \mathbf{1}_{\{\max (0, a)<b\}} .
$$

In all the following, $\Phi_{G}$ means the standard normal Gaussian distribution and one often uses the following:

$$
1-\Phi_{G}(x)=\Phi_{G}(-x) \leq \frac{1}{x \sqrt{2 \pi}} \exp -\frac{x^{2}}{2}, \quad \forall x>0 .
$$

In order to have a Lévy process with non zero jump part, let us introduce

$$
X_{t}=m t+W_{t}+\sum_{i=1}^{N_{t}} Y_{i}, \quad X_{t}^{*}=\sup _{s \leq t} X_{t},
$$

where $N$ is a Poisson process with constant positive intensity $\lambda,\left(Y_{i}, i \in \mathbb{N}^{*}\right)$ is a sequence of independent and identically distributed random variables with the distribution function $F_{Y}$ and 
the sequence of jump times of $N$ is denoted by $\left(T_{i}\right), i \geq 1$. Let $\theta$ be the shift operator and $\left(U_{t} ; \quad t \geq 0\right)$ be the $\mathbb{R}^{2}$-value process defined by

$$
U_{t}=\left(X_{t}^{*}, X_{t}\right), \quad t \geq 0
$$

The aim is to prove the theorem:

Theorem 2.1. (i) For all $t>0$, the law of the pair $\left(X_{t}^{*}, X_{t}\right)$ has a density with respect to the Lebesgue measure, denoted by $p(b, a ; t)$.

(ii) For all $t>0, a \in \mathbb{R}$ the map $h \mapsto p(a+h, a ; t)$ has a limit when $h$ goes to 0 denoted by $p(a+, a ; t)$.

(iii) Let be $\varphi: \mathbb{R}^{2} \rightarrow \mathbb{R}$ a $C_{b}^{3}$ - bounded function with a support in $\{(b, a), \quad b>0, b \geq a\}$ such that there exists $\delta>1$ satisfying $\int_{\mathbb{R}}\left|\partial_{1} \varphi(a, a)\right|^{\delta} d a<\infty$.

For any $t>0$,

$$
\begin{aligned}
& \mathbb{E}\left(\varphi\left(U_{t}\right)\right)=\varphi(0,0)+\int_{0}^{t} \mathbb{E}\left[m \partial_{2} \varphi\left(U_{s}\right)+\frac{1}{2} \partial_{22}^{2} \varphi\left(U_{s}\right)\right] d s \\
& +\int_{0}^{t} \frac{1}{2} \mathbb{E}\left[\partial_{1} \varphi\left(X_{s}, X_{s}\right) \frac{p\left(X_{s}+, X_{s} ; s\right.}{g\left(X_{s} ; s\right)}\right] d s+\lambda \int_{0}^{t} \mathbb{E}\left(\int_{\mathbb{R}}\left[\varphi\left(U_{s}(y)\right)-\varphi\left(U_{s}\right)\right] F_{Y}(d y)\right) d s .
\end{aligned}
$$

where $g(. ; s)$ is the density of the random variable $X_{s}$ and

$$
U_{s}=\left(X_{s}^{*}, X_{s}\right), \quad U_{s}(y)=\left(\max \left(X_{s}^{*}, X_{s}+y\right), X_{s}+y\right), \quad s \geq 0 .
$$

In the next sections, details of the proof of Theorem 2.1 are given.

\section{Existence of the density of the law of $\left(X_{t}^{*}, X_{t}\right)$ and its properties}

We note that

$$
X_{t}^{*}=\max \left\{\left(\sup _{u \in\left[T_{i}, \inf \left(T_{i+1}, t\right)[\right.} X_{u}, i=0, \ldots, N_{t}\right), X_{t}\right\}
$$

and use the joint density of $\left(\tilde{X}_{t}^{*}, \tilde{X}_{t}\right)$ given by (2) to show that the pair $\left(X_{t}^{*}, X_{t}\right)$ law has a density which is right continuous on the diagonal, see Proposition 3.1 below which actually is the proof of Theorem 2.1 (i) and (ii).

Proposition 3.1. (i) For all $t>0$, the law of the random vector $\left(X_{t}^{*}, X_{t}\right)$ admits a density with respect to the Lebesgue measure given by

$$
p(b, a, t)=\mathbb{E}\left(\sum_{k=0}^{N_{t}} \tilde{p}\left(b-X_{T_{k}}, a-X_{T_{k}}-Y_{k+1} \mathbf{1}_{\left\{T_{k+1} \leq t\right\}}-\left(X_{t}-X_{T_{k+1} \wedge t}\right), t \wedge T_{k+1}-T_{k}\right) \mathbf{1}_{\Delta_{k, t}}(b, a)\right)
$$

where $\tilde{p}$ is given by (2) and

$$
\Delta_{k, t}=\left\{(b, a) \mid b>\max \left(X_{T_{k}}^{*}, a+\left[X_{t \wedge T_{k+1}}-\sup _{u \in\left[T_{k+1}, t\right]} X_{u}\right] \mathbf{1}_{\left\{T_{k+1}<t\right\}}\right)\right\} .
$$


(ii) Moreover, for all $a \in \mathbb{R}, t>0$ the map $h \mapsto p(a+h, a ; t)$ has a limit when $h$ goes to 0 denoted by $p(a+, a ; t)$ and

$$
\begin{aligned}
& p(a+, a ; t)= \\
& \mathbb{E}\left(\sum_{k=0}^{N_{t}} \tilde{p}\left(\left(a-X_{T_{k}}\right)^{+}, a-X_{T_{k}}-Y_{k+1} \mathbf{1}_{\left\{T_{k+1} \leq t\right\}}-\left(X_{t}-X_{T_{k+1} \wedge t}\right), t \wedge T_{k+1}-T_{k}\right) \mathbf{1}_{a \in D_{k, t}}\right)
\end{aligned}
$$

where $D_{k, t}:=\left\{a: a \geq \max \left(X_{T_{k}}^{*}, a+\left[X_{t \wedge T_{k+1}}-\sup _{u \in\left[T_{k+1}, t\right]} X_{u}\right] \mathbf{1}_{\left\{T_{k+1}<t\right\}}\right)\right\}$

The proof of (i) relies on the following lemma:

Lemma 3.2. Almost surely, for all $t$,

$$
X_{t}^{*}=\max \left(X_{T_{k}}+\sup _{u \in\left[T_{k}, T_{k+1} \wedge t\right]}\left(\tilde{X}_{u}-\tilde{X}_{T_{k}}\right), k=0, \ldots, N_{t}\right) .
$$

Moreover, almost surely, for all t, there exists a unique $k$ denoted as $N_{t}^{*}$ such that

$$
X_{t}^{*}=X_{T_{k}}+\sup _{u \in\left[T_{k}, T_{k+1} \wedge t\right]}\left(\tilde{X}_{u}-\tilde{X}_{T_{k}}\right)
$$

Proof. Let $t$ be fixed.

(a) Note that

$$
X_{t}^{*}=\max \left\{\max \left(X_{T_{k}}+\sup _{u \in\left[T_{k}, T_{k+1} \wedge t[\right.}\left(X_{u}-X_{T_{k}}\right), \quad k=0, \ldots, N_{t}\right), X_{t}\right\} .
$$

For $k \in \mathbb{N}$, for all $u \in\left[T_{k}, T_{k+1}\left[, X_{u}-X_{T_{k}}=\tilde{X}_{u}-\tilde{X}_{T_{k}}\right.\right.$ where $\tilde{X}$ is the continuous process defined in (1), thus for $k \leq N_{t}$,

$$
\sup _{u \in\left[T_{k}, T_{k+1} \wedge t[\right.}\left(X_{u}-X_{T_{k}}\right)=\sup _{u \in\left[T_{k}, T_{k+1} \wedge t\right]}\left(\tilde{X}_{u}-\tilde{X}_{T_{k}}\right)
$$

and

$$
\max \left(X_{T_{N_{t}}}+\sup _{u \in\left[T_{N_{t}}, T_{N_{t}+1} \wedge t[\right.}\left(X_{u}-X_{T_{N_{t}}}\right), X_{t}\right)=X_{T_{N_{t}}}+\sup _{u \in\left[T_{N_{t}}, T_{N_{t}+1} \wedge t\right]}\left(\tilde{X}_{u}-\tilde{X}_{T_{N_{t}}}\right) .
$$

Plugging identities (11) and (12) in equality (10) yields (8).

(b) Let two integers $i<j$ then,

$$
X_{T_{j}}+\sup _{u \in\left[T_{j}, T_{j+1} \wedge t\right]}\left(\tilde{X}_{u}-\tilde{X}_{T_{j}}\right)=X_{T_{i}}+\left(\tilde{X}_{T_{i+1}}-\tilde{X}_{T_{i}}\right)+Y_{i+1}+\left(X_{T_{j}}-X_{T_{i+1}}\right)+\sup _{u \in\left[T_{j}, T_{j+1} \wedge t\right]}\left(\tilde{X}_{u}-\tilde{X}_{T_{j}}\right)
$$

and

$$
\begin{aligned}
& X_{T_{j}}+\sup _{u \in\left[T_{j}, T_{j+1} \wedge t\right]}\left(\tilde{X}_{u}-\tilde{X}_{T_{j}}\right)-X_{T_{i}}-\sup _{u \in\left[T_{i}, T_{i+1} \wedge t\right]}\left(\tilde{X}_{u}-\tilde{X}_{T_{i}}\right)= \\
& \left\{Y_{i+1}+\left(X_{T_{j}}-X_{T_{i+1}}\right)+\sup _{u \in\left[T_{j}, T_{j+1} \wedge t\right]}\left(\tilde{X}_{u}-\tilde{X}_{T_{j}}\right)\right\}+\left\{\left(\tilde{X}_{T_{i+1}}-\tilde{X}_{T_{i}}\right)-\sup _{u \in\left[T_{i}, T_{i+1} \wedge t\right]}\left(\tilde{X}_{u}-\tilde{X}_{T_{i}}\right)\right\} .
\end{aligned}
$$


The two following random vectors are independent:

$$
\left(\sup _{u \in\left[T_{i}, T_{i+1} \wedge t\right]}\left(\tilde{X}_{u}-\tilde{X}_{T_{i}}\right) ; \tilde{X}_{T_{i+1}}-\tilde{X}_{T_{i}}\right), Y_{i+1}+\left(X_{T_{j}}-X_{T_{i+1}}\right)+\sup _{u \in\left[T_{j}, T_{j+1} \wedge t\right]}\left(\tilde{X}_{u}-\tilde{X}_{T_{j}}\right)
$$

and the law of the vector $\left(\sup _{u \in\left[T_{i}, T_{i+1} \wedge t\right]}\left(\tilde{X}_{u}-\tilde{X}_{T_{i}}\right) ; \tilde{X}_{T_{i+1}}-\tilde{X}_{T_{i}}\right)$ admits a density with respect to the Lebesgue measure, hence the law of the random variable

$$
\sup _{u \in\left[T_{i}, T_{i+1} \wedge t\right]}\left(\tilde{X}_{u}-\tilde{X}_{T_{i}}\right)+\tilde{X}_{T_{i+1}}-\tilde{X}_{T_{i}}
$$

has a density with respect to the Lebesgue measure and is independent of

$$
Y_{i+1}+\left(X_{T_{j}}-X_{T_{i+1}}\right)+\sup _{u \in\left[T_{j}, T_{j+1} \wedge t\right]}\left(\tilde{X}_{u}-\tilde{X}_{T_{j}}\right) .
$$

Therefore, $X_{T_{j}}+\sup _{u \in\left[T_{j}, T_{j+1} \wedge t\right]}\left(\tilde{X}_{u}-\tilde{X}_{T_{j}}\right)-X_{T_{i}}-\sup _{u \in\left[T_{i}, T_{i+1} \wedge t\right]}\left(\tilde{X}_{u}-\tilde{X}_{T_{i}}\right)$ is the sum of two independent random variables, one having a density, then also has a density. So for all $t$, almost surely, whenever $i \neq j$

$$
X_{T_{j}}+\sup _{u \in\left[T_{j}, T_{j+1} \wedge t\right]}\left(\tilde{X}_{u}-\tilde{X}_{T_{j}}\right) \neq X_{T_{i}}+\sup _{u \in\left[T_{i}, T_{i+1} \wedge t\right]}\left(\tilde{X}_{u}-\tilde{X}_{T_{i}}\right)
$$

(c) Above, we can exchange $\forall t>0$ and almost surely, since the processes $\left(N_{t}, t \geq 0\right)$ and $\left(\left(\max \left(X_{T_{k}}+\sup _{u \in\left[T_{k}, T_{k+1} \wedge t\right]}\left(\tilde{X}_{u}-\tilde{X}_{T_{k}}\right)\right), \quad k \leq N_{t}\right), t \geq 0\right)$ are right continuous.

Proof. of Proposition 3.1 (i): According to Lemma 3.2, let $N_{t}^{*}$ denoting the index $k$ where the maximum below is reached,

$$
X_{t}^{*}=\max \left(X_{T_{k}}+\sup _{u \in\left[T_{k}, T_{k+1} \wedge t\right]}\left(\tilde{X}_{u}-\tilde{X}_{T_{k}}\right), k=0, \ldots, N_{t}\right) .
$$

The fact $N_{t}^{*}=k$ is equivalent to: the supremum is reached on the interval $\left[T_{k}, T_{k+1} \wedge t\right]$, actually meaning $X_{t}^{*}=\sup _{\left[T_{k}, T_{k+1} \wedge t\right]} X_{u}$ and remark that $\sup _{\left[T_{k}, T_{k+1} \wedge t\right]} X_{u} \geq X_{T_{k}}^{*} \vee \sup _{\left[T_{k+1} \wedge t, t\right]} X_{u}$.

On the interval $\left[T_{k}, T_{k+1} \wedge t\right], X_{u}=X_{T_{k}}+\tilde{X}_{u}-\tilde{X}_{T_{k}}$. Thus the following inequalities are equivalent to $N_{t}^{*}=k$ :

(a) $X_{T_{k}}+\sup _{\left[T_{k}, T_{k+1} \wedge t\right]}\left(\tilde{X}_{u}-\tilde{X}_{T_{k}}\right) \geq X_{T_{k}}^{*}$,

(b) $X_{T_{k}}+\sup _{\left[T_{k}, T_{k+1} \wedge t\right]}\left(\tilde{X}_{u}-\tilde{X}_{T_{k}}\right) \geq \sup _{\left[T_{k+1} \wedge t, t\right]} X_{u}=\left[X_{T_{k+1} \wedge t}+\sup _{\left[T_{k+1}, t\right]}\left(X_{u}-X_{T_{k+1} \wedge t}\right)\right] \mathbf{1}_{\left\{T_{k+1}<t\right\}}+X_{t} \mathbf{1}_{\left\{T_{k+1} \geq t\right\}}$.

Using $X_{T_{k+1}}=X_{T_{k}}+\tilde{X}_{T_{k+1}}-\tilde{X}_{T_{k}}+Y_{k+1}$, (b) is equivalent to

$\left.\sup _{\left[T_{k}, T_{k+1} \wedge t\right]}\left(\tilde{X}_{u}-\tilde{X}_{T_{k}}\right) \geq\left[\tilde{X}_{T_{k+1}}-\tilde{X}_{T_{k}}+Y_{k+1}+\sup _{\left[T_{k+1}, t\right]}\left(X_{u}-X_{T_{k+1}}\right)\right] \mathbf{1}_{\left\{T_{k+1}<t\right\}}\right)+\left(\tilde{X}_{t}-\tilde{X}_{T_{k}}\right) \mathbf{1}_{\left\{T_{k+1} \geq t\right\}}$.

As a conclusion we get $\left\{N_{t}^{*}=k\right\}=$

$\left.\left\{\sup _{\left[T_{k}, T_{k+1} \wedge t\right]}\left(\tilde{X}_{u}-\tilde{X}_{T_{k}}\right) \geq X_{T_{k}}^{*}-X_{T_{k}}\right\} \cap\left\{\sup _{\left[T_{k}, T_{k+1} \wedge t\right]}\left(\tilde{X}_{u}-\tilde{X}_{T_{k}}\right) \geq \tilde{X}_{t \wedge T_{k+1}}-\tilde{X}_{T_{k}}+\left[Y_{k+1}+\sup _{\left[T_{k+1}, t\right]}\left(X_{u}-X_{T_{k+1}}\right)\right] \mathbf{1}_{\left\{T_{k+1} \leq t\right\}}\right)\right\}$. 
Thus

$$
\left\{N_{t}^{*}=k\right\}=\left\{\left(\sup _{u \in\left[T_{k}, T_{k+1} \wedge t\right]}\left(\tilde{X}_{u}-\tilde{X}_{T_{k}}\right), \tilde{X}_{t \wedge T_{k+1}}-\tilde{X}_{T_{k}}\right) \in \bar{\Delta}_{k, t}\right\}
$$

where

$$
\bar{\Delta}_{k, t}=\left\{(b, a): \mid b>\max \left(X_{T_{k}}^{*}-X_{T_{k}}, a+\left[Y_{k+1}+\sup _{u \in\left[T_{k+1}, t\right]}\left(X_{u}-X_{T_{k+1}}\right)\right] \mathbf{1}_{\left\{T_{k+1} \leq t\right\}}\right)\right\} .
$$

Moreover on $\left\{k \leq N_{t}\right\}$ so on $\left\{N_{t}^{*}=k\right\} \subset\left\{k \leq N_{t}\right\}$

$$
X_{t}=X_{T_{k}}+\left(\tilde{X}_{t \wedge T_{k+1}}-\tilde{X}_{T_{k}}\right)+Y_{k+1} \mathbf{1}_{\left\{\mathbf{t} \geq \mathbf{T}_{\mathbf{k}+1}\right\}}+\left(X_{t}-X_{t \wedge T_{k+1}}\right) .
$$

Let $\Phi$ be a bounded Borel function, hence

$\mathbb{E}\left[\Phi\left(X_{t}^{*}, X_{t}\right)\right]=\mathbb{E}\left[\sum_{k=0}^{N_{t}} \Phi\left(X_{t}^{*}, X_{t}\right) \mathbf{1}_{\left\{N_{t}^{*}=k\right\}}\right]=$

$\mathbb{E}\left[\sum_{k=0}^{N_{t}} \mathbf{1}_{\left\{N_{t}^{*}=k\right\}} \Phi\left(X_{T_{k}}+\sup _{u \in\left[T_{k}, T_{k+1} \wedge t\right]}\left(\tilde{X}_{u}-\tilde{X}_{T_{k}}\right), X_{T_{k}}+\left(\tilde{X}_{t \wedge T_{k+1}}-\tilde{X}_{T_{k}}\right)+Y_{k+1} \mathbf{1}_{\left\{t \geq T_{k+1}\right\}}+\left(X_{t}-X_{t \wedge T_{k+1}}\right)\right)\right]$.

The four following random vectors are independent:

$\left(X_{T_{k}}, X_{T_{k}}^{*}\right), \quad Y_{k+1},\left(X_{t}-X_{t \wedge T_{k+1}}, \sup _{u \in\left[T_{k+1} \wedge t, t[\right.}\left(X_{u}-X_{T_{k+1} \wedge t}\right)\right),\left(\sup _{u \in\left[T_{k}, T_{k+1} \wedge t\right]} \tilde{X}_{u}-\tilde{X}_{T_{k}}, \tilde{X}_{t \wedge T_{k+1}}-\tilde{X}_{T_{k}}\right)$

and conditionally to $\sigma\left(\mathcal{F}_{T_{k}}, Y_{k+1},\left(X_{u}-X_{T_{k+1}}, \quad u \geq T_{k+1} \wedge t\right), T_{k}, T_{k+1}\right)$, the law of the random vector

$$
\left(\sup _{u \in\left[T_{k}, T_{k+1} \wedge t\right]} \tilde{X}_{u}-\tilde{X}_{T_{k}}, \tilde{X}_{t \wedge T_{k+1}}-\tilde{X}_{T_{k}}\right)
$$

has a density with respect to the Lebesgue measure given by $\tilde{p}\left(b, a, T_{k+1} \wedge t-T_{k}\right)$ where $\tilde{p}$ is defined by (2). We obtain that $\mathbb{E}\left(\Phi\left(X_{t}^{*}, X_{t}\right)\right)=$

$\int \mathbb{E}\left[\sum_{k=0}^{N_{t}} \Phi\left(X_{T_{k}}+b, X_{T_{k}}+a+Y_{k+1} \mathbf{1}_{\left\{\mathbf{t} \geq \mathbf{T}_{\mathbf{k}+1}\right\}}+\left(X_{t}-X_{T_{k+1 \wedge t}}\right)\right) \tilde{p}\left(b, a, T_{k+1} \wedge t-T_{k}\right) \mathbf{1}_{\bar{\Delta}_{k, t}}(b, a)\right] d a d b$.

The change of variable formula $v=b+X_{T_{k}}$ and $u=X_{T_{k}}+a+Y_{k+1} \mathbf{1}_{\left\{\mathbf{t} \geq \mathbf{T}_{\mathbf{k}+\mathbf{1}}\right\}}+\left(X_{t}-X_{T_{k+1 \wedge t}}\right)$ concludes the proof.

Proof of Proposition 3.1 (ii): Let $a \in \mathbb{R}, t>0$, the map

$$
h \mapsto \sum_{k=0}^{N_{t}} \tilde{p}\left(a+h-X_{T_{k}}, a-X_{T_{k}}-Y_{k+1} \mathbf{1}_{\left\{T_{k+1} \leq t\right\}}-\left(X_{t}-X_{T_{k+1} \wedge t}\right), t \wedge T_{k+1}-T_{k}\right) \mathbf{1}_{\Delta_{k, t}}(b, a)
$$

has a right limit when $h$ goes to 0 since both functions $h \mapsto \tilde{p}(a+h, a ; t)$ and $h \rightarrow \mathbf{1}_{\Delta_{k}, t}(a+h, a)$ admit a limit when $h$ decreases to 0. According to Proposition 6.2 in Appendix the family

$$
\left(\sum_{k=0}^{N_{t}} \tilde{p}\left(a+h-X_{T_{k}}, a-X_{T_{k}}-Y_{k+1} \mathbf{1}_{\left\{T_{k+1} \leq t\right\}}-\left(X_{t}-X_{T_{k+1} \wedge t}\right), t \wedge T_{k+1}-T_{k}\right) \mathbf{1}_{\Delta_{k, t}}(b, a)\right)_{h \in[0,1]}
$$


is uniformly integrable.

Then, we can exchange the limit and the expectation and $h \mapsto p(a+h, a ; t)$ has a limit when $h$ decreases to 0 and

$$
\begin{aligned}
& p(a+, a ; t)= \\
& \mathbb{E}\left(\sum_{k=0}^{N_{t}} \tilde{p}\left(\left(a-X_{T_{k}}\right)_{+}, a-X_{T_{k}}-Y_{k+1} \mathbf{1}_{\left\{T_{k+1} \leq t\right\}}-\left(X_{t}-X_{T_{k+1} \wedge t}\right), t \wedge T_{k+1}-T_{k}\right) \mathbf{1}_{a \in D_{k, t}}\right)
\end{aligned}
$$

where $D_{k, t}:=\left\{a: a \geq \max \left(X_{T_{k}}^{*}, a+\left[X_{t \wedge T_{k+1}}-\sup _{u \in\left[T_{k+1}, t\right]} X_{u}\right] \mathbf{1}_{\left\{T_{k+1}<t\right\}}\right)\right\}$

As a corollary the law of $X_{t}^{*}$ is deduced:

Corollary 3.3. For any $t>0$, the law of the random variable $X_{t}^{*}$ has a density $p^{*}(., t)$ given by

$p^{*}(b, t)=2 \mathbb{E}\left(\sum_{k=0}^{N_{t}} 2 e^{2\left(b-X_{T_{k}}\right) m} H_{m}\left[\left(C_{t, k}\right)^{+} \mathbf{1}_{T_{k+1}<t}+\left(b-X_{T_{k}}\right)+m\left(t \wedge T_{k+1}-T_{k}\right), t \wedge T_{k+1}-T_{k}\right] \mathbf{1}_{\left\{b>X_{T_{k}}^{*}\right\}}\right)$

where $H_{m}:(x, t) \rightarrow \frac{1}{\sqrt{2 \pi t}} \exp \left[-\frac{x^{2}}{2 t}\right]-m \Phi_{G}\left(-\frac{x}{\sqrt{t}}\right)$ and $C_{t, k}=\left(Y_{k+1}+\sup _{u \in\left[T_{k+1}, t\right]}\left(X_{u}-X_{T_{k+1}}\right)\right) \mathbf{1}_{\left\{T_{k+1} \leq t\right\}}$.

Proof. Let $\tilde{q}$ be the function such that $\tilde{p}(b, a,)=.\tilde{q}(b, a,.) \mathbf{1}_{b>a \vee 0}$ where

$$
\tilde{p}(b, a ; t)=\frac{2(2 b-a)}{\sqrt{2 \pi t^{3}}} \exp \left[-\frac{(2 b-a)^{2}}{2 t}+m a-m^{2} \frac{t}{2}\right] \mathbf{1}_{\{\max (0, a)<b\}} .
$$

Remark that

$$
(2 b-a)^{2}-2 m t a+m^{2} t^{2}=[a-(2 b+m t)]^{2}-4 b m t
$$

thus we obtain

$$
\tilde{q}(b, a, t)=\frac{2 e^{2 b m}}{\sqrt{2 \pi t}}\left(\frac{2 b+m t-a}{t} \exp \left[-\frac{[a-(2 b+m t)]^{2}}{2 t}\right]-m \exp \left[-\frac{[a-(2 b+m t)]^{2}}{2 t}\right]\right) .
$$

Hence, for any $A$,

$$
0 \leq \int_{-\infty}^{A} \tilde{p}(b, a, t) d a=2 e^{2 b m} \mathbf{1}_{b>0} H_{m}(x, t), x=b \wedge A-2 b-m t .
$$

Let $k$ be fixed and $P_{k}^{*}(b, t)$ be given by

$$
P_{k}^{*}(b, t):=\int_{\mathbb{R}} \tilde{p}\left(b-X_{T_{k}}, a-X_{T_{k}}-Y_{k+1} \mathbf{1}_{T_{k+1} \leq t}-\left(X_{t}-X_{T_{k+1} \wedge t}\right), t \wedge T_{k+1}-T_{k}\right) \mathbf{1}_{\Delta_{k, t}}(b, a) d a
$$

then the density of $X_{t}^{*}$ is given by

$$
p^{*}(b, t)=\mathbb{E}\left(\sum_{k=0}^{N_{t}} P_{k}^{*}(b, t)\right) .
$$


With the change of variables $u=a-X_{T_{k}}-Y_{k+1} \mathbf{1}_{T_{k+1} \leq t}-\left(X_{t}-X_{t \wedge T_{k+1}}\right)$, it follows $P_{k}^{*}(b, t):=\int_{\mathbb{R}} \tilde{p}\left(b-X_{T_{k}}, u, t \wedge T_{k+1}-T_{k}\right) \mathbf{1}_{\Delta_{k, t}}\left(b, u+X_{T_{k}}+Y_{k+1} \mathbf{1}_{T_{k+1} \leq t}+\left(X_{t}-X_{t \wedge T_{k+1}}\right)\right) d u$.

According to the definition of $\Delta_{k, t}(7)$

$$
\begin{aligned}
& \mathbf{1}_{\Delta_{k, t}}\left(b, u+X_{T_{k}}+Y_{k+1} \mathbf{1}_{\left\{T_{k+1} \leq t\right\}}+\left(X_{t}-X_{t \wedge T_{k+1}}\right)\right)= \\
& \mathbf{1}_{\left\{b>X_{T_{k}}^{*}\right\}} \mathbf{1}_{\left\{b>u+X_{T_{k}}+\left[Y_{k+1}+\sup _{u \in\left[T_{k+1}, t\right]}\left(X_{u}-X_{t}\right)\right] \mathbf{1}_{\left\{T_{k+1} \leq t\right\}}\right\}}
\end{aligned}
$$

On the event $T_{k+1} \leq t$ (id est $\left.k<N_{t}\right)$

$$
P_{k}^{*}(b, t)=\int_{\mathbb{R}} \tilde{q}\left(b-X_{T_{k}}, u, T_{k+1}-T_{k}\right) \mathbf{1}_{b>X_{T_{k}}^{*}} \mathbf{1}_{]-\infty, \min \left(b-X_{T_{k}}, b-X_{T_{k}}-C_{t, k}[\right.}(u) d u
$$

since $C_{t, k}=\left(Y_{k+1}+\sup _{u \in\left[T_{k+1}, t\right]}\left(X_{u}-X_{t}\right)\right) \mathbf{1}_{\left\{T_{k+1} \leq t\right\}}$.

And on the event $T_{k+1}>t$

$$
P_{k}^{*}(b, t)=\int_{\mathbb{R}} \tilde{q}\left(b-X_{T_{k}}, u, t-T_{k}\right) \mathbf{1}_{b>X_{T_{k}}^{*}} \mathbf{1}_{]-\infty, b-X_{T_{k}}}[(u) d u .
$$

(a) On the event $T_{k+1} \leq t$ applying (17) to $A=b-X_{T_{k}}-C_{t, k}$, with $T_{k+1}-T_{k}$ and $b-X_{T_{k}}$ instead of $t$ and $b,\left(b-X_{T_{k}}\right) \wedge A=b-X_{T_{k}}-\left(C_{t, k}\right)^{+}\left(\right.$since $\left.0 \wedge(-x)=-x^{+}\right)$so on this event

$$
P_{k}^{*}(b, t)=2 e^{2 m\left(b-X_{T_{k}}\right)} H_{m}\left[\left(C_{t, k}\right)^{+}+\left(b-X_{T_{k}}\right)+m\left(T_{k+1}-T_{k}\right), T_{k+1}-T_{k}\right] .
$$

(b) On the event $T_{k+1}>t$, applying (17) to $A=b-X_{T_{k}}$ and taking $t-T_{k}$ and $b-X_{T_{k}}$ instead of $t$ and $b$, so on this event

$$
P_{k}^{*}(b, t)=2 e^{2\left(b-X_{T_{k}}\right) m} H_{m}\left[-\left(b-X_{T_{k}}\right)-m\left(t-T_{k}\right), t-T_{k}\right] .
$$

To summarize both cases

$$
P_{k}^{*}(b, t)=2 e^{2\left(b-X_{T_{k}}\right) m} H_{m}\left[\left(C_{t, k}\right)^{+} \mathbf{1}_{\left\{T_{k+1} \leq t\right\}}+\left(b-X_{T_{k}}\right)+m\left(t \wedge T_{k+1}-T_{k}\right), t \wedge T_{k+1}-T_{k}\right]
$$

and the proof is achieved.

\section{Proof of Theorem 2.1 (iii)}

To prove the end of this theorem, we proceed as follows: we will compute $\lim _{h \rightarrow 0} h^{-1} A(t, h)=$ $a(t)$ where

$$
A(t, h):=\mathbb{E}\left[\varphi\left(U_{t+h}\right)-\varphi\left(U_{t}\right)\right] .
$$

After that, we will use [8] 11.82 p. 368: If $f$ is a function such that $f^{\prime}$ is finite everywhere and integrable, then for all $a \leq b, f(b)-f(a)=\int_{a}^{b} f^{\prime}(s) d s$. The study of $a(t):=\lim _{h \rightarrow 0} h^{-1} A(t, h)$ could prove that for all $t>t_{0}>0$,

$$
\mathbb{E}\left(\varphi\left(U_{t}\right)\right)=\int_{t_{0}}^{t} a(s) d s+\mathbb{E}\left(\varphi\left(X_{t_{0}}^{*}, X_{t_{0}}\right)\right), \quad \forall t>t_{0} .
$$


A last step will be to prove the convenient properties of the function $a$, for all $T>0$, there exist $v \in] 0,1[, \xi \geq 0$ and a constant $C$ such that

$$
\left.\left.|a(t)| \leq C\left[\frac{1}{t^{v}}+1+t^{\xi}\right], \quad \forall t \in\right] 0, T\right] .
$$

Then letting $t_{0}$ going to 0 and using the fact that $\varphi$ is continuous bounded, $X$ and $X^{*}$ are right continuous, estimation (19) and Lebesgue dominated theorem, for all $t>0$

$$
\mathbb{E}\left(\varphi\left(U_{t}\right)\right)=\int_{0}^{t} a(s) d s+\varphi(0,0) .
$$

Proof of the function $a: t \mapsto \mathbb{E}\left(\varphi\left(U_{t}\right)\right)$ admits a derivative on $] 0,+\infty[$ denoted as $a$ and satisfying (19). The idea is to split $A(t, h)$ in three parts according to the values of $N_{t+h}-N_{t}$ :

$$
A(t, h)=\sum_{i=0}^{2} A_{i}(t, h)
$$

where

$$
\begin{aligned}
& A_{i}(t, h):=\mathbb{E}\left(\left[\varphi\left(U_{t+h}\right)-\varphi\left(U_{t}\right)\right] \mathbf{1}_{\left\{N_{t+h}-N_{t}=i\right\}}\right), \quad i=0,1 \\
& \left.A_{2}(t, h):=\mathbb{E}\left(\left[\varphi\left(U_{t+h}\right)-\varphi\left(U_{t}\right)\right)\right] \mathbf{1}_{\left\{N_{t+h}-N_{t} \geq 2\right\}}\right) .
\end{aligned}
$$

Lemma 4.1. Under the hypothesis of Theorem 2.1

$$
\lim _{h \rightarrow 0} h^{-1} A_{2}(t, h)=0 .
$$

Proof. By hypothesis $\varphi$ is bounded and we get

$$
\left|A_{2}(t, h)\right| \leq 2\|\varphi\|_{\infty} \mathbb{P}\left(N_{t+h}-N_{t} \geq 2\right) \leq 2\|\varphi\|_{\infty}\left(1-e^{-\lambda h}-\lambda h e^{-\lambda h}\right) .
$$

Thus, $\lim _{h \rightarrow 0} h^{-1} A_{2}(t, h)=0$.

This lemma added to the three next propositions proves Theorem 2.1 (iii): this lemma treats the term $A_{2}(t, h)$ while Proposition 4.2 treats the term $A_{1}(t, h)$. Propositions 4.3 and 4.4 treat the term $A_{0}(t, h)$.

Proposition 4.2. Let be $\varphi: \mathbb{R}^{2} \rightarrow \mathbb{R} a C_{b}^{3}$ - bounded function. Then

$$
\lim _{h \rightarrow 0} h^{-1} A_{1}(t, h)=\lambda \mathbb{E} \int_{\mathbb{R}}\left[\varphi\left(U_{t}(y)\right)-\varphi\left(U_{t}\right)\right] F_{Y}(d y) .
$$

where $U_{t}$ is defined by (4) and $U_{t}(y)$ by (6).

This proposition gets the last term on the right hand in (5).

Proof. Introducing the term $\varphi\left(U_{t}\left(Y_{N_{t+h}}\right)\right)$, let be $A_{1}(t, h):=A_{1,1}(t, h)+A_{1,2}(t, h)$ where

$$
\begin{array}{r}
A_{1,1}(t, h)=\mathbb{E}\left(\left\{\varphi\left(U_{t}\left(Y_{N_{t+h}}\right)\right)-\varphi\left(U_{t}\right)\right\} \mathbf{1}_{\left\{N_{t+h}=N_{t}+1\right\}}\right), \\
A_{1,2}(t, h)=\mathbb{E}\left(\left\{\varphi\left(U_{t+h}\right)-\varphi\left(U_{t}\left(Y_{N_{t+h}}\right)\right)\right\} \mathbf{1}_{\left\{N_{t+h}=N_{t}+1\right\}}\right) .
\end{array}
$$


- Since $\varphi$ is $\mathcal{C}^{1}$ class with bounded derivative, Lemma 6.1 (Appendix) implies that on the event $\left\{N_{t+h}-N_{t}=1\right\}$ we have

$$
\begin{aligned}
\left|\varphi\left(U_{t+h}\right)-\varphi\left(U_{t}\left(Y_{N_{t+h}}\right)\right)\right| \leq\|\nabla \varphi\|_{\infty}\left(2 \sup _{0 \leq u \leq h}\left|\tilde{X}_{t+u}-\tilde{X}_{t}\right|+h\right) \text {. Thus } \\
h^{-1} A_{1,2}(t, h) \leq \lambda e^{-\lambda h}\|\nabla \varphi\|_{\infty}\left(2 \sup _{0 \leq u \leq h}\left|\tilde{X}_{t+u}-\tilde{X}_{t}\right|+h\right) \rightarrow 0
\end{aligned}
$$

when $h \rightarrow 0$ : Indeed, the process $\tilde{X}$ satisfies $0 \leq \tilde{X}_{h} \leq|m| h+W_{h}^{*}$ and Burkholder Davis Gundy's inequality for all $i \geq 1$ implies there exists a constant $C_{i}>0$ such that $E\left[\left(W_{h}^{*}\right)^{i}\right] \leq C_{i} h^{i / 2}$, hence for $h \leq 1$,

$$
\mathbb{E}\left(\left(\tilde{X}_{h}^{*}\right)^{i}\right) \leq C_{i} h^{i / 2}
$$

thus $E\left(\tilde{X}_{h}^{*}\right) \leq C \sqrt{h}$.

- Let us deal with $A_{1,1}(t, h)$ to show

$$
\lim _{h \rightarrow 0} h^{-1} A_{1,1}(t, h)=\lambda \mathbb{E} \int_{\mathbb{R}}\left[\varphi\left(U_{t}(y)\right)-\varphi\left(U_{t}\right)\right] F_{Y}(d y) .
$$

On the event $\left\{N_{t}=n, N_{t+h}=n+1\right\}$, the equality $U_{t}\left(Y_{N_{t+h}}\right)=U_{t}\left(Y_{n+1}\right)$ holds. The independence properties arising from the structure of the process $X$, the use of the laws of $Y_{i}, T_{n}$, the decomposition of $T_{n+1}, T_{n+1}=T_{n}+S_{n+1}$, and the conditioning to $\mathcal{F}_{T_{n}}$, yield:

$$
\begin{aligned}
& A_{1,1}(t, h)=\sum_{n=0}^{+\infty} \mathbb{E}\left[1_{\left\{T_{n} \leq t<T_{n+1} \leq t+h<T_{n+2}\right\}}\left(\varphi\left(U_{t}\left(Y_{n+1}\right)\right)-\varphi\left(U_{t}\right)\right)\right]= \\
& \sum_{n=0}^{+\infty} \mathbb{E}\left[1_{\left\{T_{n} \leq t\right\}} \int_{t-T_{n}}^{t+h-T_{n}} d s \int_{\mathbb{R}}\left(\varphi\left(U_{t}(y)\right)-\varphi\left(U_{t}\right)\right) F_{Y}(d y) \lambda e^{-\lambda\left(t+h-T_{n}\right)}\right] .
\end{aligned}
$$

By hypothesis, the function $\varphi$ is bounded and when $h$ goes to 0 , Lebesgue's dominated convergence theorem yields

$$
\lim _{h \rightarrow 0} h^{-1} A_{1,1}(t, h)=\sum_{n=0}^{+\infty} \mathbb{E}\left[\lambda e^{-\lambda\left(t-T_{n}\right)} 1_{\left\{T_{n} \leq t\right\}} \int_{\mathbb{R}}\left(\varphi\left(U_{t}(y)\right)-\varphi\left(U_{t}\right)\right) F_{Y}(d y)\right] .
$$

Since $e^{-\lambda\left(t-T_{n}\right)} \mathbf{1}_{\left\{T_{n} \leq t\right\}}=\mathbb{E}\left[\mathbf{1}_{\left\{T_{n} \leq t<T_{n+1}\right\}} / \mathcal{F}_{t}\right]$, it follows

$$
\begin{aligned}
& \lim _{h \rightarrow 0} h^{-1} A_{1,1}(t, h)=\sum_{n=0}^{+\infty} \mathbb{E}\left[\lambda 1_{\left\{T_{n} \leq t<T_{n+1}\right\}} \int_{\mathbb{R}}\left(\varphi\left(U_{t}(y)\right)-\varphi\left(U_{t}\right)\right) F_{Y}(d y)\right]= \\
& \mathbb{E}\left[\lambda \int_{\mathbb{R}}\left(\varphi\left(U_{t}(y)\right)-\varphi\left(U_{t}\right)\right) F_{Y}(d y)\right] .
\end{aligned}
$$

We now turn to the study of $h^{-1} A_{0}(t, h)$ when $h$ goes to 0 . On the event $\left\{N_{t+h}-N_{t}=0\right\}$, $T_{N_{t}}=T_{N_{t+h}}$, hence $X_{T_{N_{t+h}}}^{*}=X_{T_{N_{t}}}^{*}$ and $X_{T_{N_{t+h}}}=X_{T_{N_{t}}}$,

$$
X_{t+h}=X_{t}+\tilde{X}_{h} \circ \theta_{t}, X_{t+h}^{*}=\max \left(X_{t}^{*}, X_{t}+\tilde{X}_{h}^{*} \circ \theta_{t}\right) .
$$


Using Markov property at $t$ and the fact that the processes $N$ and $\tilde{X}$ are independent

$$
A_{0}(t, h)=e^{-\lambda h} \mathbb{E}\left(\mathbb{E}\left(\varphi\left(\max \left(x^{*}, x+\tilde{X}_{h}^{*}\right), x+\tilde{X}_{h}\right)-\varphi\left(x^{*}, x\right)\right)_{\mid x^{*}=X_{t}^{*}, x=X_{t}}\right) .
$$

Let us introduce

$$
a_{0}\left(h, x^{*}, x\right):=\mathbb{E}\left(\varphi\left(\max \left(x^{*}, x+\tilde{X}_{h}^{*}\right), x+\tilde{X}_{h}\right)-\varphi\left(x^{*}, x\right)\right) .
$$

To study the term $a_{0}\left(h, x^{*}, x\right)$, we make a Taylor expansion at a neighborhood of $\left(x^{*}, x\right)$ :

$$
\begin{aligned}
a_{0}\left(h, x^{*}, x\right): & =\partial_{2} \varphi\left(x^{*}, x\right) m h+\frac{1}{2} \partial_{22}^{2} \varphi\left(x^{*}, x\right)\left[m^{2} h^{2}+h\right]+ \\
& +\partial_{1,2}^{2} \varphi\left(x^{*}, x\right) \mathbb{E}\left(\left[\max \left(x^{*}, x+\tilde{X}_{h}^{*}\right)-x^{*}\right] \tilde{X}_{h}\right) \\
& +\frac{1}{2} \partial_{1,1}^{2} \varphi\left(x^{*}, x\right) \mathbb{E}\left(\left[\max \left(x^{*}, x+\tilde{X}_{h}^{*}\right)-x^{*}\right]^{2}\right)+R_{0}\left(h, x^{*}, x\right), \\
& +\partial_{1} \varphi\left(x^{*}, x\right) \mathbb{E}\left(\left[\max \left(x^{*}, x+\tilde{X}_{h}^{*}\right)-x^{*}\right]\right)
\end{aligned}
$$

where, using $\nabla^{i}$ the tensor of order $i$,

$$
\left|R_{0}\left(h, x^{*}, x\right)\right| \leq 4\left\|\nabla^{3} \varphi\right\|_{\infty}\left[\mathbb{E}\left(\left|\max \left(x^{*}, x+\tilde{X}_{h}^{*}\right)-x^{*}\right|^{3}\right)+\mathbb{E}\left(\left|\tilde{X}_{h}\right|^{3}\right)\right] .
$$

This allows us to write:

$$
A_{0}(t, h)=\sum_{i=1}^{3} A_{0, i}(t, h), \quad A_{0, i}(t, h):=\mathbb{E}\left(a_{0, i}\left(h,, x^{*}, x\right)_{\mid x^{*}=X_{t}^{*}, x=X_{t}}\right)
$$

where

$$
\begin{aligned}
a_{0,1}\left(h, x^{*}, x\right):= & \partial_{2} \varphi\left(x^{*}, x\right) m h+\frac{1}{2} \partial_{22}^{2} \varphi\left(x^{*}, x\right)\left[m^{2} h^{2}+h\right] \\
a_{0,2}\left(h, x^{*}, x\right):= & \partial_{1,2}^{2} \varphi\left(x^{*}, x\right) \mathbb{E}\left(\left[\max \left(x^{*}, x+\tilde{X}_{h}^{*}\right)-x^{*}\right] \tilde{X}_{h}\right) \\
& +\frac{1}{2} \partial_{1,1}^{2} \varphi\left(x^{*}, x\right) \mathbb{E}\left(\left[\max \left(x^{*}, x+\tilde{X}_{h}^{*}\right)-x^{*}\right]^{2}\right)+R_{0}\left(h, x^{*}, x\right), \\
a_{0,3}\left(h, x^{*}, x\right):= & \partial_{1} \varphi\left(x^{*}, x\right) \mathbb{E}\left(\left[\max \left(x^{*}, x+\tilde{X}_{h}^{*}\right)-x^{*}\right]\right) .
\end{aligned}
$$

Proposition 4.3. Let be $\varphi: \mathbb{R}^{2} \rightarrow \mathbb{R}$ a $C_{b}^{3}$ - bounded function. Then for any $t>0$

$$
\lim _{h \rightarrow 0} h^{-1}\left(A_{0,1}+A_{0,2}\right)(t, h)=\mathbb{E}\left(\partial_{2} \varphi\left(U_{t}\right) m+\frac{1}{2} \partial_{22}^{2} \varphi\left(U_{t}\right)\right)
$$

Proof. (a) Since $\tilde{X}$ and $\tilde{X}^{*}$ are continuous processes and $\varphi$ a three times differentiable function with bounded differential, it follows

$$
\lim _{h \rightarrow 0} h^{-1} A_{0,1}(t, h)=\mathbb{E}\left(\partial_{2} \varphi\left(U_{t}\right) m+\frac{1}{2} \partial_{22}^{2} \varphi\left(U_{t}\right)\right) .
$$


(b) The second term satisfies: Under hypothesis of Theorem 2.1,

$$
\lim _{h \rightarrow 0} h^{-1} A_{0,2}(t, h)=0 .
$$

Indeed, we first note that $\max \left(x^{*}, x+\tilde{X}_{h}^{*}\right)-x^{*}=\left(\tilde{X}_{h}^{*}-\left(x^{*}-x\right)\right)^{+} \leq \tilde{X}_{h}^{*} \mathbf{1}_{\left\{\tilde{X}_{h}^{*}>x^{*}-x\right\}}$. Using Cauchy-Schwarz inequality and (23) there exists a constant $D_{i}$ such that

$$
\mathbb{E}\left(\left[\max \left(x^{*}, x+\tilde{X}_{h}^{*}\right)-x^{*}\right]^{i}\right) \leq D_{i} h^{i / 2} \sqrt{\mathbb{P}\left(\tilde{X}_{h}^{*}>x^{*}-x\right)} .
$$

The function $\varphi$ is three times differentiable with bounded differential, we deduce from the expression of $a_{0,2}$ that there exists a constant $C>0$ such that

$$
A_{0,2}(t, h) \leq\left[\sum_{i=2}^{3}\left\|\nabla^{i} \varphi\right\|\right] C \sum_{i=2}^{3} h^{i / 2} \mathbb{E}\left(\left.\sqrt{\mathbb{P}\left(\tilde{X}_{h}^{*}>x^{*}-x\right)}\right|_{\mid x^{*}=X_{t}^{*}, x=X_{t}}\right) .
$$

The law of the pair $\left(X_{t}^{*}, X_{t}\right)$ has a density with respect to Lebesgue measure on $\mathbb{R}^{2}$, (cf. Proposition 3.1) almost surely $X_{t}^{*}>X_{t}$, it follows with Lebesgue dominated convergence Theorem

$$
\lim _{h \rightarrow 0} h^{-1} A_{0,2}(t, h)=0 .
$$

We now deal with the term $h^{-1} A_{0,3}(t, h)$.

Proposition 4.4. Let be $\varphi: \mathbb{R}^{2} \rightarrow \mathbb{R}$ a $C_{b}^{3}$ - bounded function such that there exists $\delta>1$ satisfying $\int_{\mathbb{R}}\left|\partial_{1} \varphi(a, a)\right|^{\delta} d a<\infty$. Then for any $t>0$

$$
\lim _{h \rightarrow 0} \frac{1}{h} \mathbb{E}\left(\partial_{1} \varphi\left(U_{t}\right) \mathbb{E}\left(\max \left(x^{*}, x+\tilde{X}_{h}^{*}\right)-x^{*}\right)_{x^{*}=X_{t}^{*}, x=X_{t}}\right)=\frac{1}{2} \mathbb{E}\left[\partial_{1} \varphi\left(X_{t}, X_{t}\right) \frac{p\left(X_{t}+, X_{t}, t\right)}{g\left(X_{t}, t\right)}\right] .
$$

Proof. We first need the following lemmas:

Lemma 4.5. For any $t>0$, the law of $\tilde{X}_{t}^{*}$ has the density with respect to Lebesgue measure on $\mathbb{R}$,

$$
\tilde{p}^{*}(b, t):=2\left[\frac{1}{\sqrt{2 \pi t}} \exp -\frac{(b-m t)^{2}}{2 t}-m e^{2 b m} \Phi_{G}\left(\frac{-b-m t}{\sqrt{t}}\right)\right] \mathbf{1}_{] 0,+\infty[}(b) .
$$

Remark 4.6. This result is consistent with the fact that when $m=0, \tilde{X}_{t}^{*}$ and $\left|\tilde{X}_{t}\right|$ have the same law (cf. Proposition 3.7, Revuz-Yor [7]).

Proof. This is obviously the derivative with respect to $b$ of the law provided in [4] page 147.

Lemma 4.7. Let be $h>0$ and $H(x):=\frac{1}{\sqrt{2 \pi}} e^{-\frac{x^{2}}{2}}-x \Phi_{G}(-x)$ :

$$
\begin{aligned}
& \frac{1}{2} \mathbb{E}\left(\left[\max \left(x^{*}, x+\tilde{X}_{h}^{*}\right)-x^{*}\right]\right)= \\
& -m h \int_{0}^{\infty} e^{2 b m \sqrt{h}}\left(b-\frac{\left(x^{*}-x\right)}{\sqrt{h}}\right)_{+} \Phi_{G}(-b-m \sqrt{h}) d b+\sqrt{h} H\left(\frac{\left(x^{*}-x-m h\right)}{\sqrt{h}}\right) .
\end{aligned}
$$


Proof. Recall that

$$
\mathbb{E}\left(\left[\max \left(x^{*}, x+\tilde{X}_{h}^{*}\right)-x^{*}\right]\right)=\mathbb{E}\left(\left[\tilde{X}_{h}^{*}-\left(x^{*}-x\right)\right] \mathbf{1}_{\left\{\tilde{X}_{h}^{*}>x^{*}-x\right\}}\right) .
$$

Lemma 4.5 gives the density of $\tilde{X}_{h}^{*}$ and the change of variable $b \rightarrow \sqrt{h} b$ yields

$$
\begin{aligned}
& \frac{1}{2} \mathbb{E}\left(\left[\max \left(x^{*}, x+\tilde{X}_{h}^{*}\right)-x^{*}\right]\right)= \\
& \left.\int_{\frac{x^{*}-x}{\sqrt{h}}}^{\infty} \sqrt{h}\left[b-\frac{\left(x^{*}-x\right)}{\sqrt{h}}\right]\right]_{+}\left[\frac{1}{\sqrt{2 \pi}} e^{-\frac{(b-m \sqrt{h})^{2}}{2}}-m \sqrt{h} e^{2 b m \sqrt{h}} \Phi_{G}(-b-m \sqrt{h})\right] d b .
\end{aligned}
$$

This can be written again as

$$
\begin{aligned}
& \frac{1}{2} \mathbb{E}\left(\left[\max \left(x^{*}, x+\tilde{X}_{h}^{*}\right)-x^{*}\right]\right)=\int_{\frac{x^{*}-x}{\sqrt{h}}}^{\infty} \sqrt{h}\left[b-m \sqrt{h}-\frac{\left(x^{*}-x\right)-m h}{\sqrt{h}}\right] \frac{1}{\sqrt{2 \pi}} e^{-\frac{(b-m \sqrt{h})^{2}}{2}} d b \\
& -m h \int_{\frac{x^{*}-x}{\sqrt{h}}}^{\infty} e^{2 b m \sqrt{h}}\left(b-\frac{x^{*}-x}{\sqrt{h}}\right) \Phi_{G}(-b-m \sqrt{h}) d b .
\end{aligned}
$$

The lemma is proved using the integration by parts formula and the definition of $H$.

Lemma 4.7 allows to compute $h^{-1} A_{0,3}(t, h)$ including $\mathcal{F}_{t}$-conditional expectation under the expectation:

$$
\begin{array}{r}
\frac{1}{h} \mathbb{E}\left(\partial_{1} \varphi\left(U_{t}\right) \mathbb{E}\left(\max \left(x^{*}, x+\tilde{X}_{h}^{*}\right)-x^{*}\right)_{x^{*}=X_{t}^{*}, x=X_{t}}\right)=\frac{2}{\sqrt{h}} \mathbb{E}\left(\partial_{1} \varphi\left(U_{t}\right) H\left(\frac{\left(X_{t}^{*}-X_{t}-m h\right)}{\sqrt{h}}\right)\right) \\
-2 m \mathbb{E}\left(\partial_{1} \varphi\left(U_{t}\right) \int_{0}^{\infty} e^{2 b m \sqrt{h}} \Phi_{G}(-b-m \sqrt{h})\left(b-\frac{X_{t}^{*}-X_{t}}{\sqrt{h}}\right)_{+} d b\right) .
\end{array}
$$

(a) Firstly, we show that

$$
\lim _{h \rightarrow 0}-m \mathbb{E}\left[\partial \varphi_{1}\left(U_{t}\right) \int_{0}^{+\infty} e^{2 m b \sqrt{h}} \Phi_{G}(-b-m \sqrt{h})\left(b-\frac{X_{t}^{*}-X_{t}}{\sqrt{h}}\right)_{+} d b\right]=0 .
$$

The term $\partial \varphi_{1}\left(U_{t}\right) \int_{0}^{+\infty} e^{2 m b \sqrt{h}} \Phi_{G}(-b-m \sqrt{h})\left(b-\frac{X_{t}^{*}-X_{t}}{\sqrt{h}}\right)_{+}$is uniformly bounded with respect to $h$ : Indeed, $b>0$ and let $0<h \leq 1$,

$$
0 \leq e^{2 m b \sqrt{h}} \Phi_{G}(-b-m \sqrt{h})\left(b-\frac{X_{t}^{*}-X_{t}}{\sqrt{h}}\right)_{+} \leq e^{2 m b \sqrt{h}} \Phi_{G}(-b-m \sqrt{h}) b .
$$

The function $(h, b) \mapsto e^{2 m b \sqrt{h}} \Phi_{G}(-b-m \sqrt{h}) b$ is continuous on the compact interval $\left[\begin{array}{ll}0 & 1\end{array}\right] \times\left[\begin{array}{ll}0 & 2|m|\end{array}\right]$, then it is bounded on this interval.

Now, consider $b>2|m|$. Therefore $b+m \sqrt{h}>|m|>0, \quad b-m \sqrt{h}>\frac{b}{2}$ and $\frac{b}{(b+m \sqrt{h})} \leq 2$. We use the inequality $(3): 1-\Phi_{G}(x)=\Phi_{G}(-x) \leq \frac{1}{x \sqrt{2 \pi}} \exp -\frac{x^{2}}{2}, \quad \forall x>0$, to obtain for $b>2|m|$, $h \in[0,1]$

$$
e^{2 m b \sqrt{h}} \Phi_{G}(-b-m \sqrt{h}) b \leq \frac{b}{(b+m \sqrt{h}) \sqrt{2 \pi}} e^{2 m b \sqrt{h}} e^{-\frac{(b+m \sqrt{h})^{2}}{2}} \leq \frac{2}{\sqrt{2 \pi}} e^{-\frac{b^{2}}{8}} .
$$


This implies that the term $\partial \varphi_{1}\left(U_{t}\right) \int_{0}^{+\infty} e^{2 m b \sqrt{h}} \Phi_{G}(-b-m \sqrt{h})\left(b-\frac{X_{t}^{*}-X_{t}}{\sqrt{h}}\right)_{+} d b$ is uniformly bounded by a constant. The result follows by Lebesgue dominated convergence Theorem: Indeed, almost surely $X_{t}^{*}-X_{t}>0$ and on this set, the integrand goes almost surely to 0 . .

(b) Secondly our goal is to compute the limit when $h$ goes to 0 of the term

$$
B_{1}^{*}(t, h)=E\left[\partial_{1} \varphi\left(U_{t}\right) \frac{1}{\sqrt{h}} H\left(\frac{X_{t}^{*}-X_{t}-m h}{\sqrt{h}}\right)\right] .
$$

The proof is divided into four steps.

1. Firstly, we prove that

$$
\lim _{h \rightarrow 0}\left|B_{1}^{*}(t, h)-E\left[\partial_{1} \varphi\left(U_{t}\right) \frac{1}{\sqrt{h}} H\left(\frac{X_{t}^{*}-X_{t}}{\sqrt{h}}\right)\right]\right|=0 .
$$

2. Secondly, we prove that

$$
\lim _{h \rightarrow 0}\left|B_{1}^{*}(t, h)-E\left[\partial_{1} \varphi\left(X_{t}, X_{t}\right) \frac{1}{\sqrt{h}} H\left(\frac{X_{t}^{*}-X_{t}}{\sqrt{h}}\right)\right]\right|=0 .
$$

3. Thirdly, we prove that

$$
\lim _{h \mapsto 0} B_{1}^{*}(t, h)=\frac{1}{2} \int_{\mathbb{R}} \partial_{1} \varphi(a, a ; t) p(a+, a ; t) d a .
$$

4. Finally we observe that

$$
\lim _{h \mapsto 0} B_{1}^{*}(t, h)=\frac{1}{2} E\left[\partial_{1} \varphi\left(X_{t}, X_{t}\right) \frac{p\left(X_{t}+, X_{t} ; t\right)}{g\left(X_{t}, t\right)}\right] .
$$

Step 1: The function $H$ defined by $H(x)=\frac{e^{-\frac{x^{2}}{2}}}{\sqrt{2 \pi}}-x \Phi_{G}(-x)$ is differentiable with differential given by $x \mapsto \Phi_{G}(-x)$, which is positive and bounded by 1 . Hence for all $(x, y) \in \mathbb{R}$, there exists $\lambda \in[0,1]$ such that $H(y)-H(x)=(y-x) H^{\prime}(\lambda x+(1-\lambda) y)$. Thus there exists $\lambda \in[0,1]$ such that

$$
\frac{1}{\sqrt{h}}\left[H\left(\frac{X_{t}^{*}-X_{t}}{\sqrt{h}}\right)-H\left(\frac{X_{t}^{*}-X_{t}-m h}{\sqrt{h}}\right)\right]=m H^{\prime}\left(\frac{X_{t}^{*}-X_{t}}{\sqrt{h}}+\lambda m \sqrt{h}\right)
$$

Then for $h>0$,

$$
\left|B_{1}^{*}(t, h)-E\left[\partial_{1} \varphi\left(U_{t}\right) \frac{1}{\sqrt{h}} H\left(\frac{X_{t}^{*}-X_{t}}{\sqrt{h}}\right)\right]\right| \leq|m|\left\|\partial_{1} \varphi\right\|_{\infty} E\left[\sup _{x \geq \frac{X_{t}^{*}-X_{t}}{\sqrt{h}}-|m| \sqrt{h}} H^{\prime}(x)\right] .
$$

The fact that almost surely $X_{t}^{*}-X_{t}>0$ proves that the almost sure limit of $\frac{X_{t}^{*}-X_{t}}{\sqrt{h}}-|m| \sqrt{h}$ is $+\infty$. Moreover, $H^{\prime}=\Phi_{G}$ is positive bounded and satisfies $\lim _{x \rightarrow \infty} H^{\prime}(x)=0$, so Lebesgue theorem achieves the proof of (31).

Step 2: Using regularity assumption on $\varphi$

$$
\begin{aligned}
& \left|B_{1}^{*}(t, h)-E\left[\partial_{1} \varphi\left(X_{t}, X_{t}\right) \frac{1}{\sqrt{h}} H\left(\frac{X_{t}^{*}-X_{t}}{\sqrt{h}}\right)\right]\right| \leq \\
& |m| \sqrt{h}\left\|\partial_{1} \varphi\right\|_{\infty}+\left\|\partial_{11}^{2} \varphi\right\|_{\infty} E\left[\frac{X_{t}^{*}-X_{t}}{\sqrt{h}} H\left(\frac{X_{t}^{*}-X_{t}}{\sqrt{h}}\right)\right]
\end{aligned}
$$


The function $x \mapsto x H(x)$ is bounded on $\mathbb{R}^{+}$, and $\lim _{x \rightarrow \infty} x H(x)=0$. Then, since $X_{t}^{*}-X_{t}$ law has a density and

$$
\text { almost sure } \lim _{h \rightarrow 0} \frac{X_{t}^{*}-X_{t}}{\sqrt{h}} H\left(\frac{X_{t}^{*}-X_{t}}{\sqrt{h}}\right)=0,
$$

the dominated Lebesgue Theorem yields:

$$
\lim _{h \rightarrow 0} E\left(\frac{X_{t}^{*}-X_{t}}{\sqrt{h}} H\left(\frac{X_{t}^{*}-X_{t}}{\sqrt{h}}\right)\right)=0 .
$$

The proof of (32) is achieved.

Step 3: Introducing the density of the law of $\left(X_{t}^{*}, X_{t}\right)$ according to Proposition 3.1 (i):

$$
B_{1}^{*}(t, h)=\int_{\mathbb{R}^{2}} \partial \varphi_{1}(a, a) \frac{1}{\sqrt{h}} H\left(\frac{b-a}{\sqrt{h}}\right) p(b, a ; t) d a d b+o(1) .
$$

We perform the change of variable $b=a+u \sqrt{h}$ and

$$
B_{1}^{*}(t, h)=\int_{\mathbb{R} \times \mathbb{R}^{+}} \partial_{1} \varphi(a, a) H(u) p(a+u \sqrt{h}, a, t) d a d u+o(1)
$$

Note that, for all $a>0, u>0$, according to the Proposition 3.1 (ii),

$$
\lim _{h \rightarrow 0} \partial_{1} \varphi(a, a) H(u) p(a+u \sqrt{h}, a, u)=\partial_{1} \varphi(a, a) H(u) p(a+, a, t) .
$$

Jensen inequality induces

$$
\left(B_{1}^{*}(t, h)\right)^{\delta} \leq \int_{{\mathbb{R} \times \mathbb{R}^{+}}}\left(\partial_{1} \varphi\right)^{\delta}(a, a) H^{\delta}(u) p^{\delta}(a+u \sqrt{h}, a, t) d a d u+o(1) .
$$

According to Proposition 6.2 and (39)

$$
p^{\delta}(a+u \sqrt{h}, a, t) \leq C(\delta, T, m)\left[\frac{1}{t^{v}} \frac{1}{|a+2 u \sqrt{h}+m t|^{\gamma}}+\frac{1}{t^{\beta}}+1+t^{\xi}\right] e^{6\left(m_{+}\right)^{2} \delta t}
$$

Integrability and boundedness assumptions on $\partial_{1} \varphi$ induces that for all $h$ there exists $D_{i}$ such that

$\int_{\mathbb{R} \times \mathbb{R}^{+}}\left(\partial_{1} \varphi\right)^{\delta}(a, a) H^{\delta}(u) p^{\delta}(a+u \sqrt{h}, a, t) d a d u \leq D_{1}+D_{2} \int_{\mathbb{R}^{\prime} \mathbb{R}^{+}}\left|\partial_{1} \varphi\right|^{\delta}(a, a) \frac{1}{|a+2 u \sqrt{h}+m t|^{\gamma}} H^{\delta}(u) d a d u$.

The integral with respect to $d a$ is shared in two parts factor, of $\int_{\mathbb{R}^{+}} H^{\delta}(u) d u$ :

$\int_{|a+2 u \sqrt{h}+m t| \leq 1}\left|\partial_{1} \varphi\right|^{\delta}(a, a) \frac{1}{|a+2 u \sqrt{h}+m t|^{\gamma}} d a+\int_{|a+2 u \sqrt{h}+m t|>1}\left|\partial_{1} \varphi\right|^{\delta}(a, a) \frac{1}{|a+2 u \sqrt{h}+m t|^{\gamma}} d a$.

The second term is bounded by $\int_{\mathbb{R}}\left|\partial_{1} \varphi\right|^{\delta}(a, a) d a<\infty$, the first one is bounded by

$$
\left\|\partial_{1} \varphi\right\|_{\infty} \int_{|a+2 u \sqrt{h}+m t| \leq 1} \frac{1}{|a+2 u \sqrt{h}+m t|^{\gamma}} d a=\left\|\partial_{1} \varphi\right\|_{\infty} \int_{|x| \leq 1}|x|^{-\gamma} d x=\frac{2}{1-\gamma}\left\|\partial_{1} \varphi\right\|_{\infty}
$$


Thus for all $T>0$ there exist $v \in] 0,1[, \xi \geq 0$ and a constant $C$ such that

$$
\left.\left.\sup _{h \in] 0,1]} \int_{\mathbb{R} \times \mathbb{R}^{+}}\left|\partial_{1} \varphi(a, a)\right|^{\delta} H^{\delta}(u) p^{\delta}(a+u \sqrt{h}, a, t) d a d u \leq C\left[\frac{1}{t^{v}}+1+t^{\xi}\right], \quad \forall t \in\right] 0, T\right] .
$$

The family $\left\{(a, u) \rightarrow \partial_{1} \varphi(a, a) H(u) p(a+2 u \sqrt{h}, a, t), h \in[0,1]\right\}$ is uniformly integrable with respect to Lebesgue measure $d a d u$, so we can exchange the limit and the integral:

$$
\lim _{h \rightarrow 0} B_{1}^{*}(t, h)=\int_{\mathbb{R} \times \mathbb{R}^{+}} \partial \varphi_{1}(a, a) H(u) p(a+, a ; t) d a d u .
$$

Note that $\int_{\mathbb{R}^{+}} H(u) d u=\frac{1}{2}$ ends the proof of this step.

Step 4: Propositions 4.24 .3 and 4.4, it is proved that

$a(t)=\lambda \mathbb{E} \int_{\mathbb{R}}\left[\varphi\left(U_{t}(y)\right)-\varphi\left(U_{t}\right)\right] F_{Y}(d y)+\mathbb{E}\left(\partial_{2} \varphi\left(U_{t}\right) m+\frac{1}{2} \partial_{22}^{2} \varphi\left(U_{t}\right)\right)+\frac{1}{2} \mathbb{E}\left[\partial_{1} \varphi\left(X_{t}, X_{t}\right) \frac{p\left(X_{t}+, X_{t}, t\right)}{g\left(X_{t}, t\right)}\right]$.

The two first terms are bounded, so we have only to check (19) on the third term.

Similarly to Step 3, we get

$$
\lim _{h \rightarrow 0} E\left[\left|\partial_{1} \varphi\left(U_{t}\right)\right| \frac{1}{\sqrt{h}} H\left(\frac{X_{t}^{*}-X_{t}-m h}{\sqrt{h}}\right)\right]=\int_{{\mathbb{R} \times \mathbb{R}^{+}}}\left|\partial \varphi_{1}(a, a)\right| H(u) p(a+, a ; t) d a d u .
$$

so $a \mapsto \partial \varphi_{1}(a, a) p(a+, a ; t)$ belongs to $L^{1}(\mathbb{R}, d a)$. Then, since $g(., t)$ denotes the density of the law of $X_{t} a \mapsto \partial \varphi_{1}(a, a) \frac{p(a+, a ; t)}{g(a ; t)}$ belongs to $L^{1}\left(\mathbb{R}, P_{X_{t}}\right)$.

Note that from estimation (33) for all $T>0$ there exist $v \in] 0,1[, \xi \geq 0$ and a constant $C$ such that

$$
\left.\left.\left|\frac{1}{2} \mathbb{E}\left[\partial_{1} \varphi\left(X_{t}, X_{t}\right) \frac{p\left(X_{t}+, X_{t}, t\right)}{g\left(X_{t}, t\right)}\right]\right| \leq C\left[\frac{1}{t^{v}}+1+t^{\xi}\right], \quad \forall t \in\right] 0, T\right]
$$

and the function $a$ satisfies (19).

\section{Conclusion}

The aim of this paper is to have a complete study of the law of one Lévy process $X$ and its running supremum. Recall that $X^{*}$ is not Markovian, but the pair $U:=\left(X^{*}, X\right)$ is. In the second section, we give the main result (Theorem 2.1): the density of the law of $U_{t}$, its right continuity on the diagonal and a weakly valued-measure differential equation which characterizes the law of $U_{t}$.

To complete the study of the survival probability initiated by Coutin and Dorobantu [3], as a consequence, one gives the marginal density of the law of $X_{t}^{*}$ (Corollary 3.3). A perspective could be the proof of regularity of the survival probability on $\mathbb{R}^{+} \times \mathbb{R}$, meaning

$(b, t) \rightarrow \mathbb{P}\left(\tau_{b}>t\right)=\mathbb{P}\left(X_{t}^{*} \leq b\right)=\int_{0}^{b} p^{*}(x, t) d x$.

Another perspective is to study filtering, for instance to generalize W. Ngom [6] to incomplete observation. 


\section{Appendix}

Lemma 6.1. On the event $\left\{N_{t+h}=N_{t}+1\right\},\left|U_{t}\left(Y_{N_{t+h}}\right)-U_{t+h}\right| \leq 2 \sup _{0 \leq u \leq h}\left|\tilde{X}_{t+u}-\tilde{X}_{t}\right|$.

Proof. : (a) On the event $\left\{N_{t+h}-N_{t}=1\right\}=\cup_{n}\left\{N_{t}=n, N_{t+h}-N_{t}=1\right\}$, we compute $U_{t}\left(Y_{N_{t+h}}\right)=\left(\max \left(X_{t}^{*}, X_{t}+Y_{N_{t+h}}\right) ; X_{t}+Y_{N_{t+h}}\right)$ and $U_{t+h}=\left(X_{t+h}^{*}, X_{t+h}\right)$.

(b) On the event $\left\{N_{t}=n, N_{t+h}=n+1\right\}$,

$$
U_{t}\left(Y_{N_{t+h}}\right)=\left(\max \left(X_{t}^{*}, X_{t}+Y_{n+1}\right) ; X_{t}+Y_{n+1}\right), \quad U_{t+h}=\left(X_{t+h}^{*}, X_{t+h}\right) .
$$

(c) We bound up $\left|U_{t}\left(Y_{N_{t+h}}\right)-U_{t+h}\right|$ component by component:

- Concerning the second component, on the event $\left\{N_{t}=n, N_{t+h}=n+1\right\}$, we have

$$
X_{t+h}=X_{t}+Y_{n+1}+\left(X_{t+h}-X_{t}-Y_{n+1}\right) .
$$

Since there is one only jump at time $T_{n+1}$ for the process $X$ between $t$ and $t+h$, hence $X_{t+h}-$ $X_{t}-Y_{n+1}=\tilde{X}_{t+h}-\tilde{X}_{t}$ and

$$
\left|X_{t+h}-X_{t}-Y_{n+1}\right| \mathbf{1}_{\left\{N_{t}=n, N_{t+h}=n+1\right\}} \leq \sup _{0 \leq u \leq h}\left|\tilde{X}_{t+u}-\tilde{X}_{t}\right| \mathbf{1}_{\left\{N_{t}=n, N_{t+h}=n+1\right\}} .
$$

- The first component is

$$
X_{t+h}^{*}-\max \left(X_{t}^{*}, X_{t}+Y_{n+1}\right)
$$

with

$$
\begin{aligned}
X_{t+h}^{*} & =\max \left(X_{t}^{*}, X_{t}+\sup _{t \leq u<T_{n+1}}\left(\tilde{X}_{u}-\tilde{X}_{t}\right), X_{t}+Y_{n+1}+\left(\tilde{X}_{T_{n+1}}-\tilde{X}_{t}\right)+\sup _{T_{n+1} \leq u \leq t+h}\left(\tilde{X}_{u}-\tilde{X}_{T_{n+1}}\right)\right) \\
& =\max \left(X_{t}^{*}, X_{t}+\sup _{t \leq u<T_{n+1}}\left(\tilde{X}_{u}-\tilde{X}_{t}\right), X_{t}+Y_{n+1}+\sup _{T_{n+1} \leq u \leq t+h}\left(\tilde{X}_{u}-\tilde{X}_{t}\right)\right) .
\end{aligned}
$$

(a) On the event $\left\{X_{t}^{*} \geq X_{t}+Y_{n+1}\right\}$,

$$
\begin{aligned}
& X_{t+h}^{*}-\max \left(X_{t}^{*}, X_{t}+Y_{n+1}\right)= \\
& 0 \vee\left(X_{t}+\sup _{t \leq u<T_{n+1}}\left(\tilde{X}_{u}-\tilde{X}_{t}\right)-X_{t}^{*}\right) \vee\left(X_{t}+Y_{n+1}+\sup _{T_{n+1} \leq u \leq t+h}\left(\tilde{X}_{u}-\tilde{X}_{t}\right)-X_{t}^{*}\right) .
\end{aligned}
$$

Since $X_{t} \leq X_{t}^{*}$ :

$$
X_{t}+\sup _{t \leq u \leq T_{n+1}}\left(\tilde{X}_{u}-\tilde{X}_{t}\right)-X_{t}^{*} \leq \sup _{t \leq u \leq T_{n+1}}\left(\tilde{X}_{u}-\tilde{X}_{t}\right) \leq \sup _{t \leq u \leq t+h}\left(\tilde{X}_{u}-\tilde{X}_{t}\right)
$$

and on the event $\left\{X_{t}^{*} \geq X_{t}+Y_{n+1}\right\}$

$$
X_{t}+Y_{n+1}+\sup _{T_{n+1} \leq u \leq t+h}\left(\tilde{X}_{u}-\tilde{X}_{t}\right)-X_{t}^{*} \leq \sup _{T_{n+1} \leq u \leq t+h}\left(\tilde{X}_{u}-\tilde{X}_{t}\right) \leq \sup _{t \leq u \leq t+h}\left(\tilde{X}_{u}-\tilde{X}_{t}\right) .
$$

On this event, globally

$$
0 \leq X_{t+h}^{*}-\max \left(X_{t}^{*}, X_{t}+Y_{n+1}\right) \leq \sup _{0 \leq u \leq h}\left|\tilde{X}_{t+u}-\tilde{X}_{t}\right| .
$$


(b) On the event $\left\{X_{t}^{*}<X_{t}+Y_{n+1}\right\}$, the first component is equal to

$$
\left(X_{t}^{*}-X_{t}-Y_{n+1}\right) \vee\left(\sup _{t \leq u<T_{n+1}}\left(\tilde{X}_{u}-\tilde{X}_{t}\right)-Y_{n+1}\right) \vee\left(\sup _{T_{n+1} \leq u \leq t+h}\left(\tilde{X}_{u}-\tilde{X}_{T_{n+1}}\right)\right) .
$$

On this event, the first element in (36) $\left(X_{t}^{*}-X_{t}-Y_{n+1}\right) \leq 0$ and the third one being non negative, thus the first component is $\left.\sup _{t \leq u<T_{n+1}}\left(\tilde{X}_{u}-\tilde{X}_{t}\right)-Y_{n+1}\right) \vee\left(\sup _{T_{n+1} \leq u \leq t+h}\left(\tilde{X}_{u}-\tilde{X}_{T_{n+1}}\right)\right)$.

As a conclusion, globally:

$$
\left|X_{t+h}^{*}-\max \left(X_{t}^{*}, X_{t}+Y_{n+1}\right)\right| \mathbf{1}_{\left\{N_{t}=n, N_{t+h}=n+1\right\}} \leq \sup _{t \leq u \leq t+h}\left|\tilde{X}_{u}-\tilde{X}_{t}\right| .
$$

Inequalities (34), (35) and (37) lead to the result.

\subsection{Integrability properties}

In the sequel, $P$ is the random field defined by

$$
P(b, a ; t):=\sum_{k=0}^{N_{t}} \tilde{p}\left(b-X_{T_{k}}, a-X_{T_{k}}-Y_{k+1} \mathbf{1}_{\left\{t>T_{k+1}\right\}}-\left(X_{t}-X_{\min \left(t, T_{k+1}\right)}\right) ; \min \left(t, T_{k+1}\right)-T_{k}\right)
$$

which satisfies

$$
p(b, a, t) \leq \mathbb{E}[P(b, a ; t)] .
$$

Proposition 6.2. Let $\delta \in\left[1, \frac{3}{2}\left[\right.\right.$, there exists $(v, \gamma, \beta) \in\left[0,1\left[{ }^{3}\right.\right.$ and $\xi \geq 0$, such that for all $T>0$ there exists a constant $C(\delta, T, m)$ satisfying for all $t \in] 0, T], b>\max (a, 0)$

$$
\mathbb{E}\left(P(b, a ; t)^{\delta}\right) \leq C(\delta, T, m)\left[\frac{1}{t^{v}} \frac{1}{(2 b-a+m t)^{\gamma}}+\frac{1}{t^{\beta}}+1+t^{\xi}\right] e^{6\left(m_{-}\right)^{2} \delta t}
$$

where $x_{+}=\max (x, 0)$ and $x_{-}=\max (-x, 0)$ for any $x \in \mathbb{R}$.

The proof is based on the following three lemmas.

Recall that for all $t>0$ the law of the pair $\left(\tilde{X}_{t}^{*}, \tilde{X}_{t}\right)$ has a density with respect to the Lebesgue measure given by

$$
\tilde{p}(b, a ; t)=\frac{2(2 b-a)}{\sqrt{2 \pi t^{3}}} e^{-\frac{(2 b-a)^{2}}{2 t}+m a-\frac{m^{2} t}{2}} \mathbf{1}_{\{b>\max (0, a)\}} .
$$

We have the following estimations on $\tilde{p}$.

Lemma 6.3. For all $\alpha \in] 0,1[$, there exists a constant $C(\alpha, m)$ such that for all $t>0$

$$
\tilde{p}(b, a ; t) \leq C(\alpha, m)\left[\frac{1}{t^{1-\frac{\alpha}{2}}(2 b-a+m t)^{\alpha}}+\left(m_{-}\right) \frac{1}{\sqrt{t}}\right] e^{-\frac{(2 b-a+m t)^{2}}{8 t}+6\left(m_{+}\right)^{2} t} \mathbf{1}_{\{b>\max (0, a)\}} .
$$

Proof. We factorize

$$
(2 b-a)^{2}-2 m a t+m^{2} t^{2}=[a-(2 b+m t)]^{2}-4 b m t
$$

and write

$$
\tilde{p}(b, a ; t)=\frac{2}{\sqrt{2 \pi}}\left[\frac{(2 b-a+m t)}{t \sqrt{t}}-\frac{m}{\sqrt{t}}\right] e^{-\frac{(2 b-a+m t)^{2}}{2 t}+2 b m} \mathbf{1}_{\{b>\max (0, a)\}} .
$$

Let be $C_{\beta}:=\sup _{x \in[0,+\infty[} x^{\beta} e^{-\frac{x^{2}}{4}}<+\infty$. 
- For $m \leq 0$ and since $b>0$ we obtain

$$
\tilde{p}(b, a ; t) \leq \frac{2}{\sqrt{2 \pi}}\left[\frac{C_{1+\alpha}}{t^{1-\frac{\alpha}{2}}(2 b-a+m t)^{\alpha}}+\frac{m_{-}}{\sqrt{t}}\right] e^{-\frac{(2 b-a+m t)^{2}}{4 t}} \mathbf{1}_{\{b>\max (0, a)\}},
$$

and estimation (41) for $m \leq 0$.

- For $m>0$, using (42)

$$
\tilde{p}(b, a ; t) \leq \frac{2}{\sqrt{2 \pi}}\left[\frac{C_{1+\alpha}}{t^{1-\frac{\alpha}{2}}(2 b-a+m t)^{\alpha}}\right] e^{-\frac{(2 b-a+m t)^{2}}{4 t}+2 b m} \mathbf{1}_{\{b>\max (0, a)\}} .
$$

Since

$$
\frac{(2 b-a+m t)^{2}}{8 t}-2 b m=\frac{1}{8 t}\left((2 b-a)^{2}+2(2 b-a) m t+m^{2} t^{2}-16 b m t\right),
$$

using $2 b-a \geq b$ and $m>0$,

$$
\frac{(2 b-a+m t)^{2}}{8 t}+2 b m \geq \frac{1}{8 t}\left(b^{2}+2 b m t+m^{2} t^{2}-16 b m t\right)=\frac{1}{8 t}\left((b-7 m t)^{2}-48 m^{2} t^{2}\right) \geq-6 m^{2} t^{2} .
$$

Thus

$$
\tilde{p}(b, a ; t) \leq \frac{2}{\sqrt{2 \pi}}\left[\frac{C_{1+\alpha}}{t^{1-\frac{\alpha}{2}}(2 b-a+m t)^{\alpha}}\right] e^{-\frac{(2 b-a+m t)^{2}}{8 t}+6 m^{2} t} \mathbf{1}_{\{b>\max (0, a)\}} .
$$

We obtain the estimation (41) for $m>0$.

Lemma 6.4. Let $0<\alpha<1$ there exists a constant $C(\alpha)$ such that for all $T>0, \sigma>0$

$$
\sup _{c \in \mathbb{R}} \mathbb{E}\left(|c+\sigma G|^{-\alpha} e^{-\frac{(c+\sigma G)^{2}}{2 T}}\right) \leq C(\alpha) \frac{T^{\frac{1-\alpha}{2}}}{\sigma},
$$

where $G$ is a standard Gaussian variable.

Proof. First we prove inequality (43) for $\sigma=1$. Let

$$
I(c, T):=\mathbb{E}\left(|c+G|^{-\alpha} e^{-\frac{(c+G)^{2}}{2 T}}\right)=I(c, T,+)+I(c, T,-)
$$

where

$$
I(c, T, \pm):=\mathbb{E}\left((c+G)_{ \pm}^{-\alpha} e^{-\frac{(c+G)^{2}}{2 T}}\right)
$$

Using the density of $G$

$$
I(c, T,+)=\int_{-c}^{\infty}(c+g)^{-\alpha} e^{-\frac{(c+g)^{2}}{2 T}} \frac{e^{-\frac{g^{2}}{2}}}{\sqrt{2 \pi}} d g
$$


and an integration by part

$$
I(c, T,+)=\int_{-c}^{\infty} \frac{1}{1-\alpha}\left[\frac{(c+g)^{2-\alpha}}{T}+g(c+g)^{1-\alpha}\right] e^{-\frac{(c+g)^{2}}{2 T}} \frac{e^{-\frac{g^{2}}{2}}}{\sqrt{2 \pi}} d g .
$$

Note that $x \mapsto x^{\beta} e^{-\frac{x^{2}}{4}}$ is bounded on $\mathbb{R}^{+}$by $C_{\beta}$ then

$$
I(c, T,+) \leq \int_{-c}^{\infty} \frac{1}{1-\alpha}\left[C_{2-\alpha} T^{-\frac{\alpha}{2}} \frac{e^{-\frac{(c+g)^{2}}{4 T}}}{\sqrt{2 \pi}}+C_{1} C_{1-\alpha} T^{\frac{1-\alpha}{2}} \frac{e^{-\frac{g^{2}}{4}}}{\sqrt{2 \pi}}\right] d g .
$$

Integrating with respect to $g$ yields

$$
I(c, T,+) \leq 2\left[C_{2-\alpha} T^{\frac{1-\alpha}{2}}+C_{1} C_{1-\alpha} T^{\frac{1-\alpha}{2}}\right] .
$$

Using the same lines we obtain

$$
I(c, T,-) \leq 2\left[C_{2-\alpha} T^{\frac{1-\alpha}{2}}+C_{1} C_{1-\alpha} T^{\frac{1-\alpha}{2}}\right] .
$$

Plugging inequalities (46) and (47) into (44) yields (43) for $\sigma=1$.

Replace $c$ by $\frac{c}{\sigma}$ and $T$ by $\frac{T}{\sigma^{2}}$ yields (43).

Lemma 6.5. For all $\alpha<1, \beta<1, \delta>0, T>0$ there exists a constant $C(\alpha, \beta, \delta, T)$ such that for all $t \in] 0, T]$,

$$
\mathbb{E}\left(\mathbf{1}_{\left\{N_{t}>0\right\}}\left(N_{t}+1\right)^{\delta}\left[T_{N_{t}}^{-\beta}\left(t-T_{N_{t}}\right)^{-\alpha}+\sum_{k=0}^{N_{t}-1}\left(t-T_{k+1}\right)^{-\alpha}\left(T_{k+1}-T_{k}\right)^{-\beta}\right]\right) \leq C(\alpha, \beta, \delta, T) t^{1-\alpha-\beta} .
$$

Proof. Let $S(1, \alpha, \beta, \delta, t)$ be the random variable defined by

$$
S(1, \alpha, \beta, \delta, t):=\mathbb{E}\left(\mathbf{1}_{\left\{N_{t}>0\right\}}\left(N_{t}+1\right)^{\delta}\left[T_{N_{t}}^{-\beta}\left(t-T_{N_{t}}\right)^{-\alpha}\right]\right) .
$$

Note that $\left(T_{k+1}-T_{k}\right)_{k \geq 0}$ are independent variables, identically distributed and $T_{1}$ follows an exponential distribution with parameter $\lambda$.

Then, $S(1, \alpha, \beta, \delta, t)=\sum_{n=1}^{\infty} S(1, \alpha, \beta, \delta, t ; n)$ where for $n \geq 1$ :

$$
\begin{aligned}
& S(1, \alpha, \beta, \delta, t ; n) \\
= & (n+1)^{\delta} \int_{[0, \infty[n+1}\left(u_{1}+\ldots+u_{n}\right)^{-\beta}\left(t-u_{1}-\ldots-u_{n}\right)^{-\alpha} \lambda^{n+1} e^{-\lambda\left(u_{1}+\ldots+u_{n+1}\right)} \mathbf{1}_{\left\{u_{1}+. .+u_{n} \leq t \leq u_{1}+\ldots+u_{n+1}\right\}} d u_{1} \ldots d u_{n}-
\end{aligned}
$$

We integrate with respect to $u_{n+1}$ between $t-u_{1}-\ldots-u_{n}$ and infinity $S(1, \alpha, \beta, \delta, t ; n)=\lambda^{n}(n+1)^{\delta} e^{-\lambda t} \int_{[0, \infty[n}\left(u_{1}+\ldots+u_{n}\right)^{-\beta}\left(t-u_{1}-\ldots-u_{n}\right)^{-\alpha} \mathbf{1}_{\left\{u_{1}+. .+u_{n} \leq t\right\}} d u_{1} \ldots d u_{n}$.

We perform the change of variable $t v_{i}=u_{1}+\ldots+u_{i}, i=1, \ldots, n$

$$
S(1, \alpha, \beta, \delta, t ; n)=\lambda^{n} t^{n-\alpha-\beta} e^{-\lambda t} \int_{[0,1]^{n}}\left(v_{n}\right)^{-\beta}\left(1-v_{n}\right)^{-\alpha} \mathbf{1}_{\left\{v_{1}<.<v_{n} \leq 1\right\}} d v_{1} \ldots d v_{n} .
$$


We integrate with respect to $v_{1}, \ldots, v_{n-1}$

$$
\begin{aligned}
S(1, \alpha, \beta, \delta, t ; n) & =\frac{(n+1)^{\delta} \lambda^{n} t^{n-\alpha-\beta}}{(n-1) !} e^{-\lambda t} \int_{0}^{1}\left(v_{n}\right)^{-\beta+n-1}\left(1-v_{n}\right)^{-\alpha} d v_{n} \\
& =\frac{(n+1)^{\delta} \lambda^{n} t^{n-\alpha-\beta}}{(n-1) !} e^{-\lambda t} B(n-\beta, 1-\alpha)
\end{aligned}
$$

The radius of convergence of entire series $\left(\sum_{n \geq 1} \frac{(n+1)^{\delta} \lambda^{n} t^{n-1}}{(n-1) !} B(n-\beta, 1-\alpha)\right)$ is infinite, so the series is continuous on $\mathbb{R}$ and then bounded on $[0, T]$. There exists a constant $C_{1}(\alpha, \beta, \delta, T)$ such that for all $t \in] 0, T]$ adding inequalities (51) yields

$$
S(1, \alpha, \beta, \delta, t) \leq C_{1}(\alpha, \beta, \delta, T) t^{1-\alpha-\beta} .
$$

Let $S(2, \alpha, \beta, \delta, t)$ be the random variable defined by

$$
S(2, \alpha, \beta, \delta, t):=\mathbb{E}\left(\mathbf{1}_{\left\{N_{t}>0\right\}}\left(N_{t}+1\right)^{\delta} \sum_{k=0}^{N_{t}-1}\left[\left(t-T_{k+1}\right)^{-\beta}\left(T_{k+1}-T_{k}\right)^{-\alpha}\right]\right) .
$$

Note that $\left(T_{k+1}-T_{k}\right)_{k \geq 0}$ are independent variables, identically distributed and $T_{1}$ follows an exponential distribution with parameter $\lambda$.

Then,

$$
S(2, \alpha, \beta, \delta, t)=\sum_{n=1}^{\infty} \sum_{k=0}^{n-1} S(2, \alpha, \beta, \delta, t ; n, k)
$$

where for $n \geq 1,0 \leq k \leq n-1$

$$
\begin{aligned}
& S(2, \alpha, \beta, \delta, t ; n, k) \\
= & (n+1)^{\delta} \int_{[0, \infty[n+1}\left(t-u_{1}-\ldots-u_{k+1}\right)^{-\beta}\left(u_{k+1}\right)^{-\alpha} \lambda^{n+1} e^{-\lambda\left(u_{1}+\ldots+u_{n+1}\right)} \mathbf{1}_{\left\{u_{1}+. .+u_{n} \leq t \leq u_{1}+\ldots+u_{n+1}\right\}} d u_{1} . . d u_{n+1} .
\end{aligned}
$$

We integrate with respect to $u_{n+1}$ between $t-u_{1}-. . u_{n}$ and infinity

$$
S(2, \alpha, \beta, \delta, t ; n, k)=(n+1)^{\delta} e^{-\lambda t} \lambda^{n} \int_{[0, \infty[n}\left(t-u_{1}-\ldots-u_{k+1}\right)^{-\beta}\left(u_{k+1}\right)^{-\alpha} \mathbf{1}_{\left\{u_{1}+. .+u_{n} \leq t\right\}} d u_{1} \ldots d u_{n} .
$$

We perform the change of variable $t v_{i}=u_{1}+\ldots+u_{i}, i=1, \ldots, n$

$$
S(2, \alpha, \beta, \delta, t ; n, k)=(n+1)^{\delta} t^{n-\alpha-\beta} e^{-\lambda t} \lambda^{n} \int_{[0,1]^{n}}\left(1-v_{k+1}\right)^{-\beta}\left(v_{k+1}-v_{k}\right)^{-\alpha} \mathbf{1}_{\left\{v_{1}<. .<v_{n} \leq 1\right\}} d v_{1} \ldots d v_{n} .
$$

We integrate with respect to $v_{1}, \ldots, v_{k}$ and $v_{k+2}, \ldots, v_{n}$

$$
\begin{aligned}
S(2, \alpha, \beta, \delta, t ; n, k) & =\frac{(n+1)^{\delta} t^{n-\alpha-\beta} e^{-\lambda t} \lambda^{n}}{k !(n-k) !} \int_{[0,1]^{2}}\left(1-v_{k+1}\right)^{-\beta+n-k}\left(v_{k+1}-v_{k}\right)^{-\alpha+k} d v_{k} d v_{k+1} \\
& \leq \frac{(n+1)^{\delta} t^{n-\alpha-\beta} e^{-\lambda t} \lambda^{n}}{n !} \frac{k !(n-k) !}{n !} \max (1, B(1-\beta, 1-\alpha))
\end{aligned}
$$

since

$0 \leq \int_{[0,1]^{2}}\left(1-v_{k+1}\right)^{-\beta+n-k}\left(v_{k+1}-v_{k}\right)^{-\alpha+k} d v_{k} d v_{k+1} \leq \max \left(1, \int_{[0,1]^{2}}\left(1-v_{k+1}\right)^{-\beta}\left(v_{k+1}-v_{k}\right)^{-\alpha} d v_{k} d v_{k+1}\right)$. 
Then, adding inequalities ( 55 ) from $k=0$ to $n-1$

$$
\sum_{k=0}^{n} S(2, \alpha, \beta, \delta, t ; n, k) \leq \max \left(1, \frac{\Gamma(-\alpha+1) \Gamma(-\beta+1)}{\Gamma(-\alpha-\beta+1)},\right) \frac{(n+1)^{\delta} 2^{n} t^{n-\alpha-\beta} e^{-\lambda t} \lambda^{n}}{n !} .
$$

The radius of convergence of entire series $\left(\sum_{n \geq 1} \frac{(n+1)^{\delta} \lambda^{n} 2^{n} t^{n-1}}{(n) !}\right)$ is infinite, so the series is continuous on $\mathbb{R}$ and then bounded on $[0, T]$ : there exists a constant $C_{2}(\alpha, \beta, \delta, T)$ such that for all $t \in] 0, T]$ and adding inequalities (56) yields

$$
S(2, \alpha, \beta, \delta, t) \leq C_{2}(\alpha, \beta, \delta, T) t^{1-\alpha-\beta} .
$$

Inequality (48) is a consequence of inequalities (52) and (57).

Proof. of the proposition: recall

$$
P(b, a ; t):=\sum_{k=0}^{N_{t}} \tilde{p}\left(b-X_{T_{k}}, a-X_{T_{k}}-Y_{k+1} \mathbf{1}_{\left\{t>T_{k+1}\right\}}-\left(X_{t}-X_{\min \left(t, T_{k+1}\right)}\right) ; \min \left(t, T_{k+1}\right)-T_{k}\right) .
$$

- Note that from Lemma 6.3 , there exists a constant $C(\alpha, m)$ such that

$$
P(b, a ; t) \mathbf{1}_{\left\{N_{t}=0\right\}} \leq C(\alpha, m)\left[\frac{1}{t^{1-\frac{\alpha}{2}}(2 b-a+m t)^{\alpha}}+(m)_{+} \frac{1}{\sqrt{t}}\right] e^{6\left(m_{+}\right)^{2} t} \mathbf{1}_{\{b>\max (0, a)\}}
$$

Taking $\xi=0, \beta=\frac{\delta}{2}$. Since $\delta \in\left[1, \frac{3}{2}\left[\right.\right.$ then $2\left(1-\frac{1}{\delta}\right)<\frac{1}{\delta}$. If $\alpha=1-\frac{1}{2 \delta}$ then $\gamma:=\alpha \delta=$ $\delta-\frac{1}{2}<1$ and $v:=\delta\left(1-\frac{\alpha}{2}\right)<1$. Then, using $(x+y)^{\delta} \leq 2^{\delta-1}\left[x^{\delta}+y^{\delta}\right]$ there exists a constant $C_{0}(\delta)$ such that for all $t>0, b>\max (a, 0)$

$$
\mathbb{E}\left(P(b, a ; t)^{\delta} \mathbf{1}_{\left\{N_{t}=0\right\}}\right) \leq C_{0}(\delta)\left[\frac{1}{t^{v}} \frac{1}{(2 b-a+m t)^{\gamma}}+\frac{1}{t^{\beta}}\right] e^{6\left(m_{+}\right)^{2} t \delta} .
$$

- Using estimation (41) and development (38)

$$
\begin{aligned}
& P(b, a ; t):=\sum_{k=0}^{N_{t}} \tilde{p}\left(b-X_{T_{k}}, a-X_{T_{k}}-Y_{k+1} \mathbf{1}_{\left\{t>T_{k+1}\right\}}-\left(X_{t}-X_{\min \left(t, T_{k+1}\right)}\right) ; \min \left(t, T_{k+1}\right)-T_{k}\right), \\
& P(b, a ; t) \mathbf{1}_{\left\{N_{t}>0\right\}} \leq C(\alpha, m) \mathbf{1}_{\left\{N_{t}>0\right\}} \sum_{k=0}^{N_{t}} P_{k}(b, a, t) \text { where } \\
& P_{k}(b, a ; t) \leq\left[\frac{1}{\left(t \wedge T_{k+1}-T_{k}\right)^{1-\alpha / 2} Z_{k}(t)}+\frac{m_{-}}{\sqrt{t \wedge T_{k+1}-T_{k}}}\right] e^{-\frac{Z_{k}(t)^{2}}{8 t}} e^{6\left(m_{-}\right)^{2}\left(\min \left(t, T_{k+1}\right)-T_{k}\right)} \\
& \text { and } Z_{k}(t):=\left(2 b-a-X_{T_{k}}+Y_{k+1} \mathbf{1}_{\left\{t>T_{k+1}\right\}}+X_{t}-X_{t \wedge T_{k+1}}+m\left(T_{k+1} \wedge t-T_{k}\right) .\right.
\end{aligned}
$$

We define a family $\left(C_{k}(t), \sigma_{k}(t), S_{k}(t), G_{k}(t)\right)_{k \in \mathbb{N} \cup\{0\}}$ by

- $\left(C_{k}(t), \sigma_{k}(t), S_{k}(t), G_{k}(t)\right)=(0,0,0,0)$ for $k>N_{t}$,

- for $k<N_{t}$ :

$$
C_{k}(t)=2 b-a-X_{T_{k}}+\sum_{i=k+1}^{N_{t}} Y_{i}+m\left(t-T_{k+1}\right)+m\left(T_{k+1}-T_{k}\right)
$$




$$
\sigma_{k}(t)=\sqrt{t-T_{k+1}} ; S_{k}(t)=T_{k+1}-T_{k} ; G_{k}(t)=\frac{B_{t}-B_{T_{k+1}}}{\sqrt{t-T_{k+1}}}
$$

- for $k=N_{t}$

$C_{k}(t)=2 b-a-\sum_{i=0}^{N_{t}} Y_{i}-m T_{N_{t}}+m\left(t-T_{N_{t}}\right) ; \sigma_{k}(t)=\sqrt{T_{N_{t}}} ; \quad S_{k}(t)=t-T_{N_{t}}, ; G_{k}(t)=\frac{B_{T_{N_{t}}}}{\sqrt{T_{N_{t}}}}$.

Then, from estimation (41) and the definition of $\left(C_{k}(t), \sigma_{k}(t), S_{k}(t), G_{k}(t)\right)$

$$
P(b, a ; t) \mathbf{1}_{\left\{N_{t} \neq 0\right\}} \leq \mathbf{1}_{\left\{N_{t} \neq 0\right\}} \sum_{k=0}^{N_{t}}\left[\frac{1}{S_{k}^{1-\frac{\alpha}{2}}\left(C_{k}+\sigma_{k} G_{k}\right)^{\alpha}}+(m)_{-} \frac{1}{\sqrt{S_{k}}}\right] e^{6\left(m_{+}\right)^{2} t} \mathbf{1}_{\{b>\max (0, a)\}}
$$

Note that $S_{k} \leq t$ and using Jensen inequality

$$
P(b, a ; t)^{\delta} \mathbf{1}_{\left\{N_{t} \neq 0\right\}} \leq \mathbf{1}_{\left\{N_{t} \neq 0\right\}}\left(N_{t}+1\right)^{\delta} \sum_{k=0}^{N_{t}}\left[\frac{1}{S_{k}^{\delta\left(1-\frac{\alpha}{2}\right)}\left(C_{k}+\sigma_{k} G_{k}\right)^{\alpha} \delta}+(m)_{-}^{\delta} \frac{1}{\sqrt{S_{k}}}\right] e^{6 \delta\left(m_{+}\right)^{2} t} \mathbf{1}_{\{b>\max (0, a)\}}
$$

Conditionally to $\left\{N_{t} \neq 0\right\}$, the random variable $G_{k}$ are independent of $C_{k}, \sigma_{k}$ and $S_{k}$ and their law is the standard Gaussian distribution. Using Lemma 6.5 for $\sigma=\sigma_{k}$ and $T=S_{k}$ yields there exists a constant $C(\alpha, \delta)$ such that

$$
\begin{aligned}
& \mathbb{E}\left(P(b, a ; t)^{\delta} \mathbf{1}_{\left\{N_{t} \neq 0\right\}}\right) \leq \\
& C(\alpha, \delta) \mathbb{E}\left(\mathbf{1}_{\left\{N_{t} \neq 0\right\}}\left(N_{t}+1\right)^{\delta} \sum_{k=0}^{N_{t}-1}\left[\frac{1}{\left(T_{k+1}-T_{k}\right)^{\delta-\frac{1}{2}} \sqrt{t-T_{k+1}}}+(m)_{-}^{\delta} \frac{1}{\sqrt{t-T_{k+1}}}\right]\right) e^{6 \delta\left(m_{+}\right)^{2} t} \mathbf{1}_{\{b>\max (0, a)\}} \\
& \left.+C_{(} \alpha, \delta\right) \mathbb{E}\left(\mathbf{1}_{\left\{N_{t} \neq 0\right\}}\left(N_{t}+1\right)^{\delta}\left[\frac{1}{T_{N_{t}}^{\delta-\frac{1}{2}} \sqrt{t-T_{N_{t}}}}+(m)_{-}^{\delta} \frac{1}{\sqrt{T_{N_{t}}}}\right]\right) e^{6 \delta\left(m_{+}\right)^{2} t} \mathbf{1}_{\{b>\max (0, a)\}} .
\end{aligned}
$$

According to Lemma 6.5, there exists a constant $C(\alpha, \delta, T)$ such that for all $t \in] 0, T]$

$$
\mathbb{E}\left(P(b, a ; t)^{\delta} \mathbf{1}_{\left\{N_{t} \neq 0\right\}}\right) \leq C(\alpha, \delta, T)\left[t^{1-\delta}+t^{\frac{1-\delta}{2}}\right] e^{6 \delta\left(m_{+}\right)^{2} t} \mathbf{1}_{\{b>\max (0, a)\}} .
$$

Adding inequalities (58) and (59) yields inequality (40).

\section{References}

[1] D. Applebaum (2009), Lévy Processes and Stochastic Calculus, second edition, Cambridge university press.

[2] P. Carr and L. Cousot A PDE approach to jump diffusions, Quantitative Finance, Vol. 11, $N^{0} 1$, pages 33-52, Taylor \& Francis, 2011.

[3] L. Coutin, D. Dorobantu First passage time law for some Lévy processes with compound Poisson: existence of a density, Vol. 17, $N^{0}$ 4, pp 1127-1135, Bernoulli Society for Mathematical Statistics and Probability, 2011. 
[4] M. Jeanblanc, M.Yor, M. Chesney (2009) Mathematical Methods for Financial Markets, Springer.

[5] S. G. Kou and H. Wang First passage time of a jump diffusion process Adv. Appl. Prob. 35, pp. 504-531, 2013.

[6] W. Ngom Conditional Law of the Hitting Time for a Lévy Process in Incomplete Observation Journal of Mathematical Finance, vol. 5, number 5, pages 505-524, 2015.

[7] D. Revuz and M. Yor Continuous Martingales and Brownian Motion, Vol. 293, Springer Science \& Business Media, 2013.

[8] Titchmarsh "The Theory of Functions, 2d Edition, Oxford 1939.

[9] M. Veillette, and M. S. Taqqu Using differential equations to obtain joint moments of firstpassage times of increasing Lévy processes, Statistics \& Probability Letters, Vol. 80, $N^{0} 7$, pp. 697-705, Elsevier, 2010. 